\title{
Digitalisierung für Mensch und Organisation im Aftersales
}

\section{Ein Beispiel der Grimme Landmaschinenfabrik $\mathrm{GmbH} \& \mathrm{Co} . \mathrm{KG}$}

\author{
Marcus Pier, Carina Siedler, Stephanie Dupont, Klaus J. Zink \\ und Jan C. Aurich
}

\section{Zusammenfassung}

Der Wandel vom reinen Maschinenbauunternehmen zum Anbieter von Produkt-ServiceSystemen stellt Landmaschinenhersteller wie die GrimmeLANDMASCHINENFABRIK GMвH \& Co. KG vor große Herausforderungen. Um diesen Wandel vollziehen zu können, sind ein gut ausgebildetes Fachpersonal, kurzfristige Ersatzteilverfügbarkeit sowie moderne Systeme für Dokumentation und Kommunikation notwendig. Im Rahmen einer Pilotanwendung im Verbundforschungsprojekt InAsPro wird der Fokus auf den Personalentwicklungsprozess und damit auf ein gut ausgebildetes Fachpersonal gelegt. Durch ein Kursverwaltungssystem wird ein individueller Kompetenzaufbau der eigenen Servicemitarbeiter und der Servicetechniker der GRIMME-Servicepartner angestrebt. Zur nachhaltigen und individuellen Weiterbildung wird eine Kompetenzmatrix verwendet, welche zusätzlich um Lehrmodule ergänzt wird und so eine Sensibilisierung der Menschen im Bereich der Digitalisierung von Sach- und Serviceprodukten fördert.

M. Pier

Grimme Landmaschinenfabrik GmbH \& Co. KG, Kaiserslautern, Deutschland

E-Mail: m.pier@grimme.de

C. Siedler $(\square) \cdot$ J. C. Aurich

Technische Universität Kaiserslautern, Kaiserslautern, Deutschland

E-Mail: carina.siedler@mv.uni-kl.de; jan.aurich@mv.uni-kl.de

S. Dupont · K. J. Zink

Institut für Technologie und Arbeit (ITA), Kaiserslautern, Deutschland

E-Mail: stephanie.dupont@ita-kl.de; klaus.zink@ita-kl.de

(C) Der/die Autor(en) 2021

T. Jeske und F. Lennings (Hrsg.), Produktivitätsmanagement 4.0, ifaa-Edition, https://doi.org/10.1007/978-3-662-61584-3_8 


\subsection{Vorstellung der Grimme Landmaschinenfabrik GmbH \& Co. KG}

Die Landwirtschaft steht in den nächsten Jahren vor tiefgreifenden Veränderungen. Grund dafür ist die wachsende Weltbevölkerung, welche bei gleichbleibender Versorgungsfläche auch in Zukunft ernährt werden muss. Die hierfür notwendige Produktivitätssteigerung kann u. a. durch datenbasierte Serviceprodukte ermöglicht werden, indem z. B. Landwirte bei der Ernte durch individuelle Handlungsempfehlungen unterstützt werden [19]. Voraussetzung für die Nutzung dieser Serviceprodukte sind Landmaschinen, welche Daten erheben und verarbeiten können. Diese Landmaschinen werden nicht nur von nationalen Anbietern, sondern zunehmen auch von internationaler Konkurrenz gebaut, die ebenfalls Ersatzteile und Serviceprodukte anbieten. Für die GrimmeLandmaschinenfabrik GmbH \& Co. KG (im Folgenden: Grimme) [10] wird es daher die zentrale Herausforderung der nächsten fünf Jahre sein, das Unternehmen von einem Maschinenbauunternehmen zum Anbieter von Produkt-Service Systemen weiterzuentwickeln und dadurch Meilensteine im Aftersales zu setzen. Zentrale Elemente des Wandels sind dabei gut ausgebildetes Fachpersonal, kurzfristige Ersatzteilverfügbarkeit sowie Daten und moderne System für Dokumentation und Kommunikation. Um die genannte Zielsetzung zu erreichen, wurden für GRIMME sieben Zukunftstrends abgeleitet, die im Bereich Aftersales konsequent umgesetzt werden (Abb. 8.1), wobei ein besonderer Fokus auf der Kundenzufriedenheit liegt.

\section{Geschichte der Grimme Landmaschinefabrik GmbH \& Co. KG}

Bereits im Jahre 1861 fand die Grundsteinlegung von GrIMME am heutigen Standort in Damme statt. Die Schmiede wurde durch den Urgroßvater des heutigen Firmeneigentümers gegründet. Bis in die 1930er Jahre war die Familie Grimme einzig mit dem alltäglichen Schmiedebetrieb beauftragt [12].

Erst in den folgenden Jahren widmete sich GRIMME der Kartoffeltechnik und entwickelte einen Kartoffelablagekorb, welcher für damalige Verhältnisse in einer großen Stückzahl von circa 1000 Stück produziert wurde. In den folgenden Jahren wurde die Kartoffelerntetechnik weiter optimiert, bis im Jahre 1956 der erste in Serie gefertigte, gezogene Kartoffelroder der Landmaschinenfabrik auf dem Markt erschien. Im Jahr 1966 legte das Unternehmen mit dem ersten gezogenen Kartoffelroder mit hydraulischen Funktionen einen europaweiten Meilenstein. Durch diesen strategisch wichtigen Schritt konnte Grimme neue Absatzmärkte in Europa gewinnen. Nur drei Jahre später entwickelte Franz Grimme Senior den ersten Kartoffelroder in Form eines Selbstfahrers mit eigenem Antrieb. 


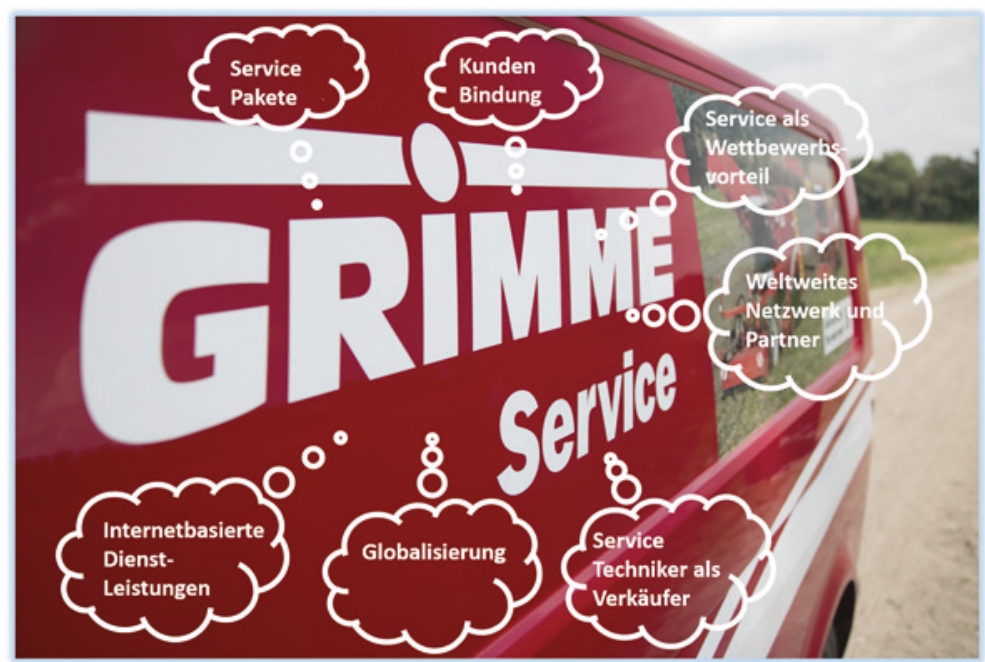

Abb. 8.1 Zukunftstrends des Grimme Aftersales

1980 stieg Franz Grimme Junior in das Unternehmen ein. In den folgenden Jahren wurde das Produktprogramm des Unternehmens weiter ausgebaut. So gelang GRIMME 1999 der Einstieg in die Legetechnik, Pflegetechnik sowie in die Einlagerungstechnik mit eigenen entwickelten Maschinen. Dieser Schritt ließ GRIMME zu einem Komplettanbieter, einem sog. Full Liner [3], in der Kartoffeltechnik heranwachsen.

Im Jahr 2003 gelang es dem Unternehmen durch den selbstfahrenden Rübenvollernter Maxtron 620 mit einem neuen Gesamtkonzept in die Rübentechnik einzusteigen [11]. 2013 übernahm GRIMmE einen im Markt fest etablierten Rübentechnikhersteller. Mithilfe der durch die Übernahme neu erworbenen Kompetenzen will sich GRIMME auch in der Rübentechnik zum Full Liner entwickeln.

Der Hauptsitz von Grimme befindet sich seit Firmengründung in Damme (auch Werk 1 genannt) und wurde im Jahr 2000 um das Werk 2 in Rieste erweitert, welches für die Montage von selbstfahrenden Arbeitsmaschinen zuständig ist. Insgesamt arbeitet an diesen zwei Standorten eine Stammbelegschaft von 1450 Mitarbeitern. Hinzu kommen noch ca. 125 Auszubildende und Studenten, die ihren zukünftigen Beruf in 14 verschiedenen Ausbildungs- und Studienrichtungen erlernen.

Die GrimmeLandmaschinenfabrik GmbH \& Co. KG befindet sich heute in vierter Generation in Familienbesitz (Abb. 8.2). Dabei entwickelte sich GrIMME im Lauf der Jahrzehnte vom Spezialisten in der Kartoffeltechnik für Feld und Halle zum weltweit agierenden Anbieter innovativer Kartoffel-, Gemüse- und Zuckerrübentechnik. Neben der Entwicklung und Fertigung der Maschinen übernimmt GRIMME zusammen mit weltweiten Servicepartnern auch deren Betreuung während der Nutzungszeit. Im Jahr 2017 erwirtschaftete das Unternehmen einen Umsatz von über 300 Mio. €. 


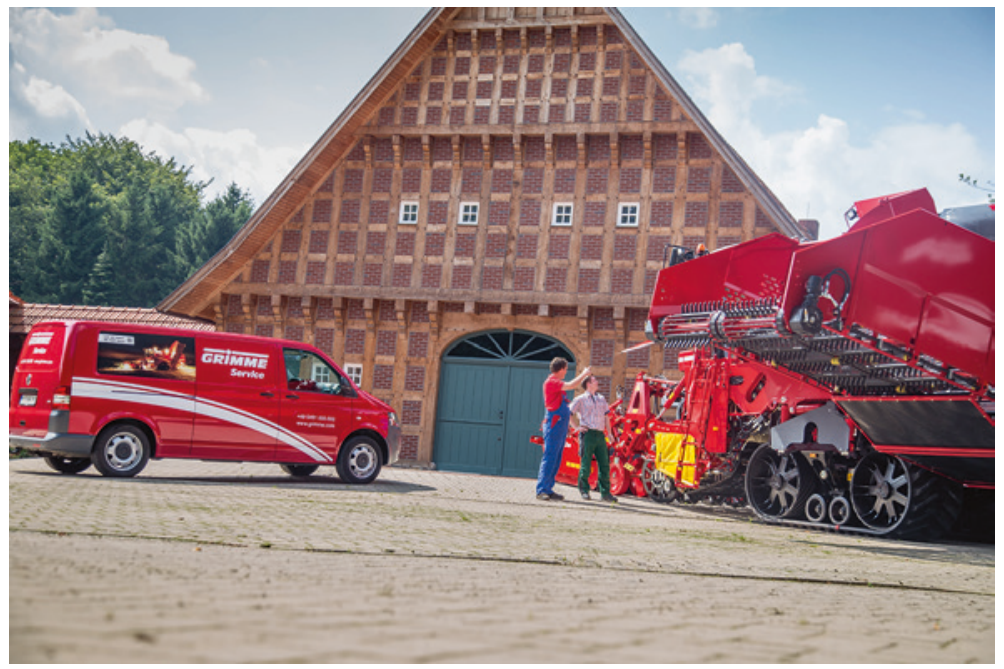

Abb. 8.2 Grimme Landmaschinenfabrik $\mathrm{GmbH}$

Neben der Landmaschinenfabrik besteht die Grimme Gruppe noch aus fünf weiteren Tochtergesellschaften. Dazu gehört der Rübentechnikhersteller KLEINE, die Gemüsetechnikspezialisten AsA-LIFT, das Bänderwerk Ricon, der Polyurethan (PU)-Gussspezialist InTERNORM, der Nordamerikanische Kartoffeltechnik Full Liner SPUDNIK sowie viele weitere Vertriebsgesellschaften, die die GrimmE- TechNIK erfolgreich in 120 Länder vertreiben. Weltweit beschäftigt die GrimmE Gruppe damit über 2000 Menschen, die Entwicklung, Produktion und Verkauf von über 150 verschiedenen Maschinentypen vorantreiben [10].

Um einen flächendeckenden Service anbieten $\mathrm{zu}$ können, arbeitet GRIMME mit Partnern vor Ort zusammen. Diese müssen nicht nur in Beratung und Verkauf kompetente Ansprechpartner sein, sondern auch in den Bereichen Service, GrImME-Originalteile und Gebrauchtmaschinenvermarktung die beste Lösung für den Kunden bieten. Dieser Anspruch wird insbesondere durch ausgesuchte GRIMMEPremium-Partner verwirklicht, die jederzeit engen Kontakt zum Stammsitz in Damme pflegen. Der enge Austausch zwischen Kunde, Vertriebspartner und Werksbeauftragtem ist wichtig, denn nur so kann GRIMME an Kundenbedarfen orientierte innovative und leistungsstarke Produkte entwickeln. Die Vertriebspartner werden in intensiven Trainings in Theorie und Praxis geschult, sodass im Bedarfsfall schnelle Unterstützung für den Kunden gewährleistet ist. Für diesen Zweck hat GrimmE eine Academy mit zwei Standorten in Damme und Cappeln gegründet. 


\subsection{Ausgangssituation der Pilotanwendung im Aftersales}

\section{Motivation}

Der GRIMmE-Aftersales erbringt weltweit Serviceleistungen für die Kunden. Allerdings gerät diese Tätigkeit in Zukunft verstärkt unter Druck durch äußere Einflüsse, wie bspw. die sich verändernden Lebensbedingungen und Ernährungsgewohnheiten sowie Umweltkatastrophen. Um die bestmögliche Kundenzufriedenheit $\mathrm{zu}$ erreichen, werden heute nicht nur die Maschinen als „Produkt“ verkauft, sondern es werden Produkt-Service Systeme (PSS) entwickelt, die die Einsätze der Produkte begleiten. PSS bestehen dabei aus Sachprodukten, Serviceprodukten, Netzwerken von Akteuren und einer unterstützenden Infrastruktur [21]. Zur Realisierung solcher Systemlösungen müssen drei Aspekte berücksichtigt werden:

- Sehr gut ausgebildete Beschäftigte mit klarer Verantwortung

- Sofortige Teile-Verfügbarkeit im Service

- Up-to-date Systeme für Kommunikation, Organisation und Dokumentation [23]

Zur Erreichung dieser Ziele nimmt GRIMME am Verbundforschungsprojekt ,Integrierte Arbeitssystemgestaltung in digitalisierten Produktionsunternehmen“ (InAsPro) [37] teil. Hierbei werden wesentliche Themen der Digitalisierung bei GRIMME, insbesondere hinsichtlich Beschäftigter und Arbeitsorganisation, adressiert.

\section{Das Projekt InAsPro}

Das Ziel des Projekts InAsPro ist es, ein modulares Transformationskonzept für die bedarfsgerechte Implementierung von Digitalisierungstechnologien in Unternehmen zu entwickeln. Dabei wird ein partizipativer Ansatz verfolgt, der mitarbeiterbezogene, technische und organisatorische Faktoren bei der Auswahl und Integration von Digitalisierungstechnologien für die Produktlebenszyklusphasen Entwicklung, Fertigung, Montage und Aftersales berücksichtigt. Das Transformationskonzept besteht aus vier Einzelkonzepten: Arbeitssystemanalyse, Digitalisierungsgrad, Digitalisierungsstrategie und Digitalisierung des Arbeitssystems (Abb. 8.3) [32].

Im Konzeptbestandteil „Arbeitssystemanalyse“ wird ein Arbeitssystem im Unternehmen hinsichtlich seiner vorhandenen Digitalisierungstechnologien analysiert und zukünftige Potenziale identifiziert. Aktuelle und zukünftige Digitalisierungstechnologien werden unter Berücksichtigung ihrer Einsetzbarkeit in den verschiedenen Produktlebenszyklusphasen in einem Technologieatlas systematisch beschrieben [30, 36]. Das zweite Konzeptbestandteil ist ein neu entwickeltes Reifegradmodell zur Ermittlung des Digitalisierungsgrades eines Arbeitssystems innerhalb verschiedener Produktlebenszyklusphasen. Im dritten Konzeptbestandteil werden Digitalisierungsstrategien abgeleitet und mit der Unternehmensstrategie abgestimmt. Im letzten Konzeptbestandteil liegt der 


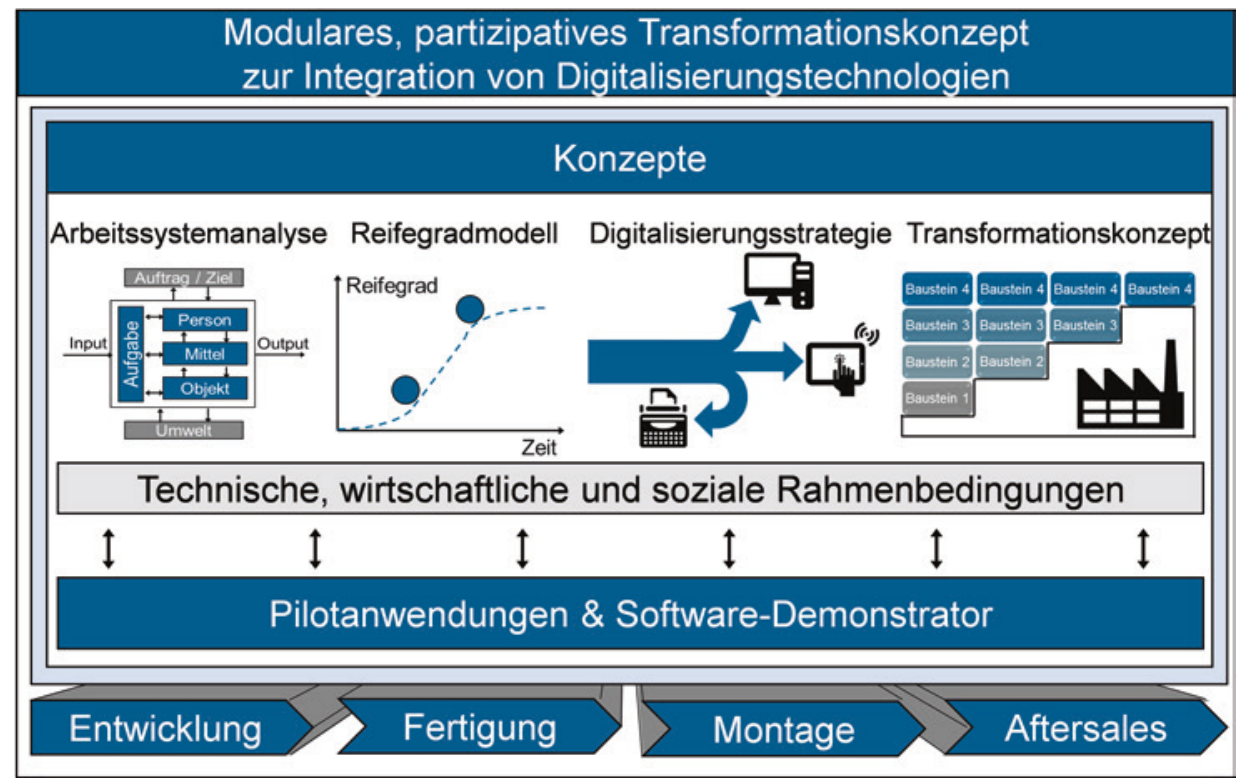

Abb. 8.3 Rahmenwerk des Projekts InAsPro

Fokus auf den operativen Umsetzungsmaßnahmen. Hier wird gezeigt, wie ein Unternehmen ausgewählte Digitalisierungstechnologien implementieren kann [28].

Diese vier Einzelkonzepte bilden zusammen das modulare Transformationskonzept. Die theoretische Entwicklung des Konzepts wird von vier industriellen Pilotanwendungen unter Berücksichtigung der vier Produktlebenszyklusphasen begleitet [9]. Zusätzlich wird das entwickelte Transformationskonzept in einem Softwaredemonstrator umgesetzt und auf der Homepage des Projekts (www.inaspro.de) zur Verfügung gestellt, sodass die Ergebnisse auch nach Abschluss des Forschungsprojekts angewendet werden können $[6,7]$.

\section{Die Pilotanwendung „Digitalisierung für Mensch und Organisation im Aftersales“6 bei Grimme}

In den letzten Jahren wurden bei GRIMME insbesondere im Aftersalesbereich wesentliche digitale Systeme entwickelt und eingeführt. Hierzu zählt z. B. ein Online-Portal zur Dokumentationsbereitstellung und Abarbeitung von Serviceanfragen mithilfe eines Workflowmanagements. Parallel dazu wurde weltweit eine digitale Servicelösung für alle Außendienstmitarbeiter eingeführt, die mithilfe von Tablets genutzt werden kann. Eine umfassende Qualifikation der Servicetechniker stellt dabei ein wesentliches Element für die Erreichung einer hohen Servicequalität dar. Durch die GrIMME ACADEMY und den bei der Industrie- und Handelskammer (IHK) Lüneburg eingerichteten Ausbildungsgang „Servicetechniker für Bau- und Landmaschinen“ verfügen die 
Servicetechniker von GRIMME über fachlich-technische, organisatorische sowie soziale Kompetenzen zur Bewältigung der sich stets wandelnden Kundenanforderungen.

Die Personalentwicklung von GRIMME ermittelt auf Grundlage der Unternehmensbedarfe berufsbegleitende und arbeitsplatznahe Aus- und Weiterbildungsmöglichkeiten für die Mitarbeiter und leitet geeignete Maßnahmen und Strategien ab, die einen Beitrag zur Erreichung der Unternehmensziele liefern können. Aktuell werden Schulungsangebote unternehmensübergreifend und in vier Kompetenzstufen angeboten. Dabei findet zwar die Ausrichtung auf eine Zielgruppe statt, jedoch soll die Berücksichtigung der Entwicklungspotenziale und -wünsche jedes einzelnen Teilnehmers noch nachhaltiger angegangen werden.

Im Rahmen von InAsPro betrachtet GRIMME vor allem die Rolle der Mitarbeiter in digitalisierten Produktionssystemen. Es soll untersucht werden, wie sich die Anforderungen an die Mitarbeiter im Kontext der zunehmenden Digitalisierung in cyber-physischen Produktionssysteme (CPPS) und weiteren Unternehmensbereichen wandeln. CPPS adressieren die enge Verbindung eingebetteter Systeme zur Überwachung und Steuerung physikalischer Vorgänge mit Sensoren und Aktuatoren über Kommunikationseinrichtungen mit den globalen digitalen Netzen [4]. Hierzu kann GRIMME auf Expertise aus dem Bereich der Mitarbeiterschulung zurückgreifen und die Ergebnisse des Forschungsprojekts in das Aus- und Weiterbildungsangebot einfließen lassen. Im Rahmen der Pilotanwendung werden die durch Digitalisierung ausgelösten Anforderungen gespiegelt sowie die erarbeiteten Lösungen angewendet und validiert.

Die Pilotanwendung im Projekt InAsPro setzt bei den Personalentwicklungsprozessen im Aftersales an. Dabei bildet das Aftersales die Schnittstelle zwischen dem Hersteller (hier: GRIMME) und den Endanwender von Anbaugeräten für die Landwirtschaft (Bodenbearbeitung, Saat- und Erntetechnik für Kartoffeln, Rüben und Gemüse). Der Fokus liegt dabei auf dem Kompetenzaufbau der eigenen Servicetechniker sowie der Servicetechniker, der in Vertrag stehenden Händler. Im Zentrum der angestrebten Digitalisierungslösungen steht eine personenbezogene Datenerhebung und -auswertung sowie eine resultierende empfehlungsbasierte Weiterbildungsmatrix. In der Pilotanwendung werden modulare Qualifikationsbausteine entwickelt und im genannten Funktionsbereich zur Anwendung kommen. Die Personalentwicklung soll zunächst unternehmensintern an der Zielgruppe „Aftersales“ stärker bedarfs- und händlerorientiert ausgerichtet werden [35].

Ein wesentlicher Entwicklungsansatz sind definierte und planbare Kompetenzentwicklungsschritte unter Berücksichtigung der Schulungshistorie, die individuell auf die Bedürfnisse einzelner Mitarbeiter angepasst werden können. Daraus entsteht ein Weiterbildungskonzept für Mitarbeiter, das sowohl Kompetenzen zur Einführung und Umsetzung von Digitalisierungstechnologien, als auch zum erfolgreichen Arbeiten betrachtet. Die dazu notwendigen Kompetenzprofile für Aftersales-Mitarbeiter werden entwickelt und Möglichkeiten der systematischen Abbildung dieser Kompetenzprofile geschaffen. Daraus werden Entwicklungsvorschläge für die Mitarbeiter abgeleitet, um zukunftsfähige Kompetenzbilder realisieren zu können. Dies geschieht mit einer im 
Projekt definierten digitalen Kompetenzmatrix, die Handlungsfelder mit Entwicklungsmöglichkeiten der Mitarbeitenden abgleicht. Sie wird genutzt, um planbare Kompetenzentwicklungsschritte zu ermitteln, die zum einen die Schulungshistorie und zum anderen die individuellen Bedürfnisse der Mitarbeiter berücksichtigen. Daraus leitet sich ein Weiterbildungskonzept für Mitarbeiterkompetenzen in digitalisierten Arbeitssystemen ab, das zusätzlich durch Lehrmodule ergänzt wird. Die Lehrmodule werden zur Sensibilisierung von Mitarbeitern im Bereich der Digitalisierung von Sach- und Serviceprodukten (z. B. Händler- und Anwenderschulung, Instandhaltungsangebote) genutzt, wobei explizit auch arbeitswissenschaftliche Grundlagen integriert werden.

Der Schwerpunkt der Pilotanwendung liegt daher in der Produktlebenszyklusphase Aftersales im Bereich Mensch und Organisation. Der Dimension Technik kommt eine unterstützende Funktion zu, da er für die Umsetzung der digitalen Kompetenzmatrix benötigt wird Abb. 8.4 Die Umsetzung der Pilotanwendung erfordert eine enge Zusammenarbeit zwischen verschiedenen Abteilungen und Akteuren Abb. 8.5. Während die Personalentwicklung die zentrale Organisationseinheit für die Umsetzung der Pilotanwendung im Aftersales darstellt, arbeitet eine Vielzahl weiterer Abteilungen dem Projekt zu: Die Abteilung Finanzen erstellt für die Pilotanwendung eine Kostenübersicht sowie eine Kostenkalkulation. Die IT-Abteilung entwickelt das IT-System, das sowohl Wissensstände erhebt, Schulungsangebote sichtbar macht und eine Anbindung an die vorhandene IT-Infrastruktur ermöglicht. Zur Erfüllung dieser Funktionen benötigt das IT-System personenbezogene Daten. Daher prüft zunächst die Rechtsabteilung, welche personenbezogenen Daten verwendet werden können, bevor der Betriebsrat entscheidet, ob diese im Unternehmen auch tatsächlich erhoben und verarbeitet werden dürfen.

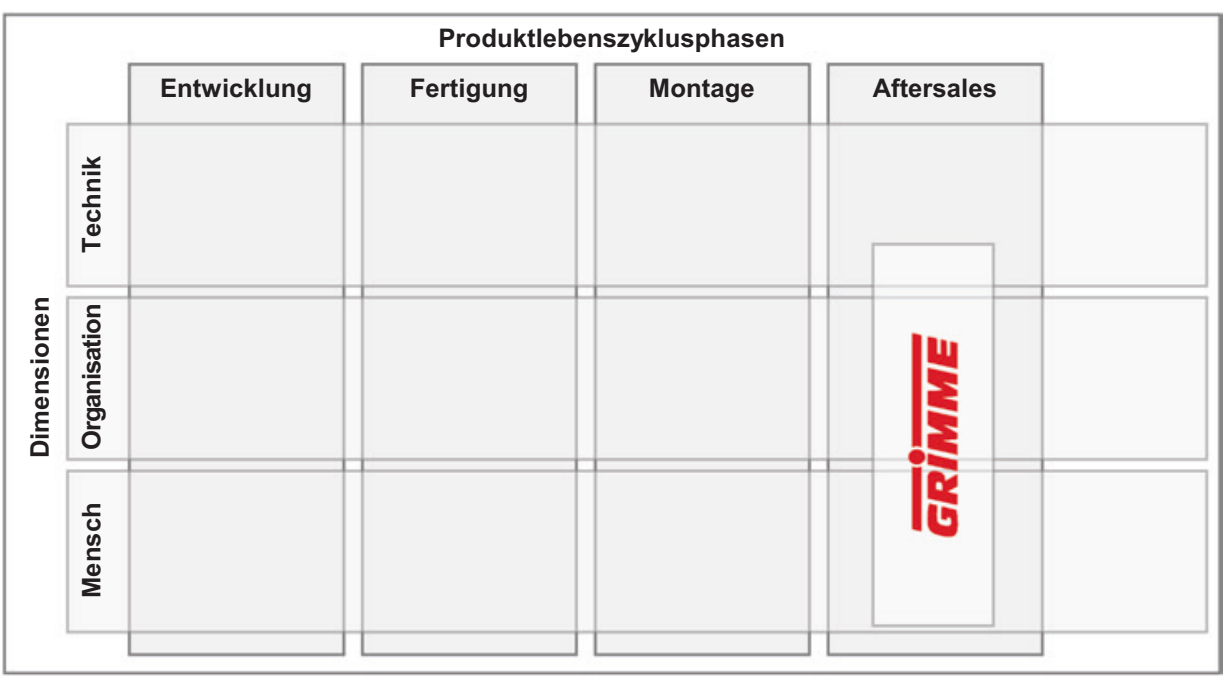

Abb. 8.4 Einordnung der Pilotanwendung in die Produktlebenszyklusphasen und Dimensionen 


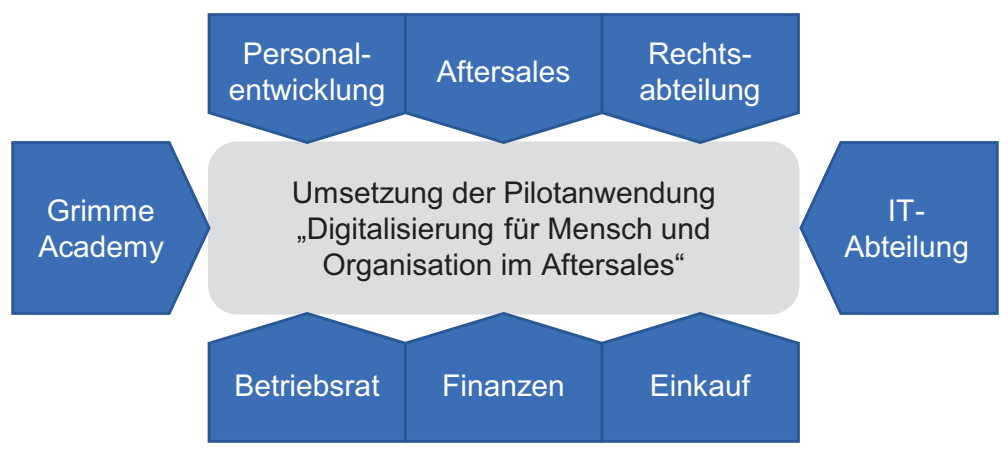

Abb. 8.5 Beteiligte Abteilungen und Akteure an der Umsetzung der Pilotanwendung

Sollte die unternehmensinterne IT-Abteilung nicht ausreichend Kapazitäten für die Entwicklung bereitstellen können, wird der Einkauf involviert. Dieser führt gegebenenfalls Verhandlungen mit externen Anbietern der IT-Systeme bzw. externen Entwickeln durch. Das resultierende IT-System wird in der GRIMME ACADEMY genutzt, um passende Weiterbildungsangebote auswählen und die Kompetenzen der Mitarbeiter weiterentwickeln zu können.

\section{Beschreibung des Arbeitssystems im Rahmen der Pilotanwendung}

Die Umsetzung der geplanten Pilotanwendungen geht mit erheblichen Veränderungen für den Bereich Aftersales einher. Um die Auswirkungen der Veränderung für das Unternehmen analysieren und darauf aufbauend bestmöglich gestalten zu können, bietet sich eine Betrachtung des Arbeitssystems an. Ein Arbeitssystem ist ein soziotechnisches System, das das Zusammenwirken von verschiedenen Systemelementen beschreibt, die zur Erledigung einer Arbeitsaufgabe notwendig sind (Abb. 8.6). Das zentrale Element ist dabei der Mitarbeiter bzw. die Arbeitsperson, die auf Grundlage von Input, Zielvorgaben und Umwelteinflüssen unter Zuhilfenahme von Arbeitsmitteln und Arbeitsobjekten eine Arbeitsaufgabe erfüllt, wodurch Output erzeugt wird [15]. Die Wechselwirkungen dieser Elemente werden in Abb. 8.6 aufgezeigt.

Die Arbeitsperson ist der Mitarbeiter, der eine oder mehrere Tätigkeiten auf Grundlage seiner Motivation, Leistungsbereitschaft und individueller Kompetenzen, durchführt. Hierfür nutzt er Input, der aus Informationen, Daten oder Material bestehen kann, sowie vorhandene Zielvorgaben. Die hierfür verwendeten Arbeitsmittel sind Werkzeuge, die zur Erledigung der Aufgabe notwendig sind, einschließlich Hardware und Software, Maschinen, Fahrzeuge oder Einrichtungsgegenstände. Das zu bearbeitende Arbeitsobjekt kann als physischer Gegenstand oder beispielsweise auch in Form von Daten vorliegen. In jedem Fall wird das Arbeitsobjekt durch die Arbeitsperson verändert, um einen Output zu erzielen. Der Output ist das Arbeitsergebnis, das auf Basis von Art, Qualität und Quantität beschrieben werden kann. Grundlage für diesen Prozess bilden 


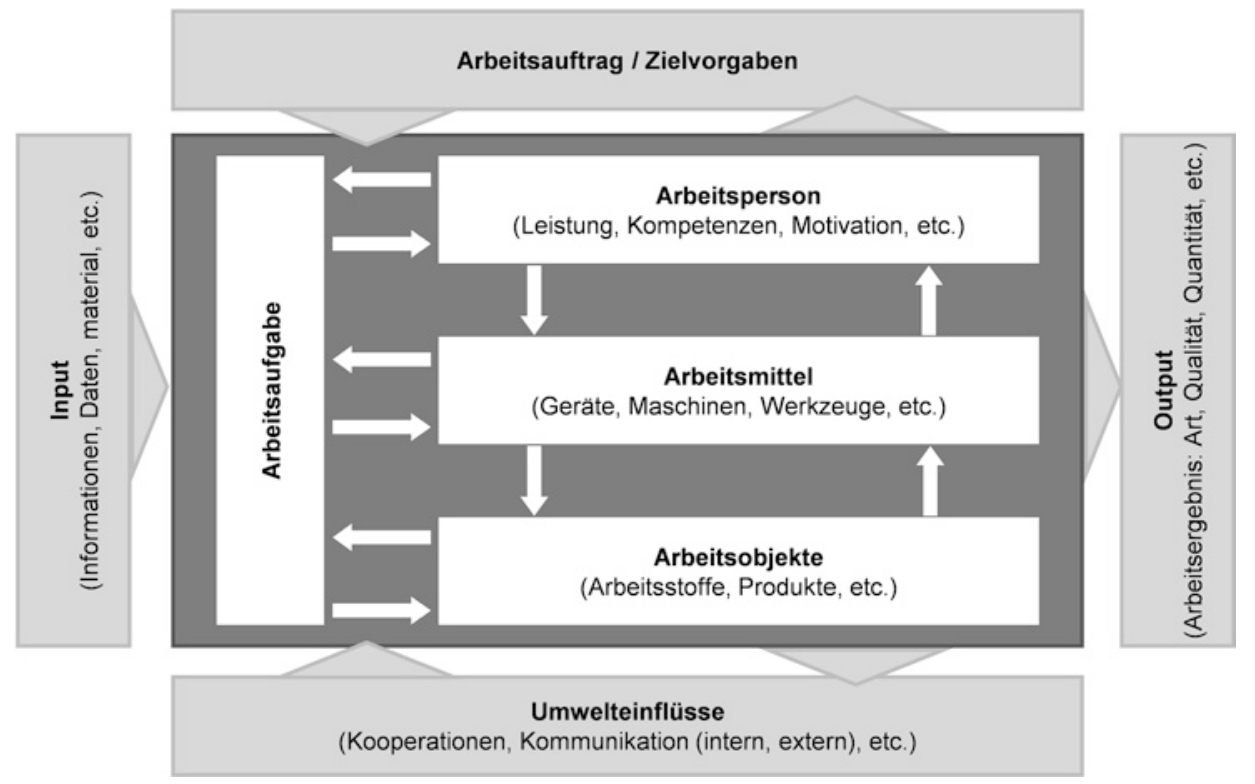

Abb. 8.6 Elemente des Arbeitssystems nach Schlick et al. [26]

Umwelteinflüsse, die die Art und Weise der Aufgabenerledigung beeinflussen, wie beispielsweise Kooperationsformen im Unternehmen oder Kommunikationsmöglichkeiten [26].

Um notwendige Entwicklungsschritte für die Planung von Veränderungsprozessen identifizieren zu können, bietet die Betrachtung von Arbeitssystemen die Möglichkeit, Veränderungen für alle Systemelemente zu beschreiben. Ausgehend von einem Istzustand werden Entwicklungsschritte unternommen, die zu einem Zielzustand führen.

$\mathrm{Da}$ es sich bei der hier vorgestellten Pilotanwendung um ein Digitalisierungsvorhaben handelt, ist der Zielzustand ein digitalisiertes Arbeitssystem. Änderungen von Elementen im Arbeitssystem führen durch die aufgezeigte enge Vernetzung der Elemente zwangsläufig auch zu Veränderungen von anderen Systemelementen. Obwohl die Pilotanwendung auf die Entwicklung und Einführung eines Kursverwaltungssystems (KVS), also die Veränderung des Arbeitsmittels, abzielt, können weitreichende Veränderungen von Arbeitsaufgabe, Arbeitsmittel, Arbeitsobjekt sowie Input und Output festgestellt werden. Umwelteinflüsse, beispielsweise der Schulungsbedarf von Monteuren sowie rechtliche Rahmenbedingungen, unterscheiden sich zwischen Ausgangssituation und Zielzustand nicht und können damit vernachlässigt werden. Auch die Arbeitsperson mit ihrer Zielvorgabe ändert sich nicht. Das Ziel ist weiterhin der Kompetenzaufbau der Mitarbeiter im Bereich Aftersales und dieses Ziel soll von der Arbeitsperson, in diesem Fall dem Personalentwickler in Zusammenarbeit mit der jeweiligen Fachabteilung, erreicht werden. 
Die größten Veränderungen zwischen Istzustand und Zielzustand zeigen sich bei der Arbeitsaufgabe. In der Ausgangssituation wurden Personalentwicklungsmaßnahmen konzipiert und den jeweiligen Mitarbeitergruppen zugeordnet. Zukünftig werden darüber Wissensstände der jeweiligen Mitarbeiter ermittelt und die Mitarbeiter in Fähigkeitsklassen eingeordnet werden. Aufbauend darauf wird der individuelle Schulungsbedarf anhand einer Qualifizierungsmatrix ermittelt und gezielte Schulungsmaßnahmen durchgeführt werden. Um diese weiterführenden Arbeitsaufgaben erreichen zu können, wird der Arbeitsperson ein neues Arbeitsmittel zur Verfügung gestellt. Ursprünglich konnten Informationen über das unternehmensinterne ERP-System eingesehen werden, das mit einem Dokumentenmanagementsystem verknüpft war, in dem die Schulungsangebote hinterlegt sind. Aufbauend darauf wurde im Rahmen der Pilotanwendung ein KVS entwickelt, das interne Informationsquellen aus dem vorhandenen ERP-System mit externen Informationen von Monteuren zusammenführen kann und so die Schulungsplanung für die Arbeitsperson erleichtert. Dadurch verändert sich auch das Arbeitsobjekt, da nicht mehr einzelne Qualifizierungsmaßnahmen geplant werden, sondern Kompetenzprofile der Aftersales-Mitarbeiter bearbeitet werden. Die Veränderung wirkt sich auf Input und Output aus, da nun nicht mehr vorhandene Schulungsangebote aus einem Schulungskatalog als Inputinformationen genutzt werden, sondern strategische Kompetenzbedarfe, die Schulungshistorie von Mitarbeitern und Bedarfsmeldungen im KVS hinterlegt sind. Dadurch kann ein spezialisierter Output generiert werden. Schulungsangebote werden nicht mehr als E-Mail oder in Papierform verschickt und Rückmeldungen müssen nicht mehr händisch erfasst und verwaltet werden. Vielmehr können Entwicklungsmöglichkeiten individualisiert elektronisch ermittelt, dem Mitarbeiter zugewiesen und durch den Vorgesetzten freigegeben werden.

\subsection{Vorgehensweise zur Zielerreichung für Unternehmen}

Um die gesetzten Ziele im Rahmen der Pilotanwendung zu erreichen, werden die genannten Einzelkonzepte angewandt. Zunächst wird der Digitalisierungsgrad des Unternehmens mit dem im Projekt InAsPro entwickelten Reifegradmodell erhoben, bevor die Digitalisierungsstrategie entwickelt und passende Digitalisierungslösungen identifiziert werden. Anschließend wird der Istzustand bei GRIMME detailliert aufgenommen und die Umsetzung zur Implementierung der gewählten Digitalisierungslösung geplant (Abschn. 8.3.1). Die Einzelkonzepte können im entwickelten Softwaredemonstrator durchlaufen werden, welcher auf der Homepage des Projekts zur Verfügung steht [37]. Abschließend erfolgt die Realisierung bei GrImme vor Ort (Abschn. 8.3.2). Unterstützt wird diese Umsetzung durch Regelmeetings mit den beteiligten Projektpartnern (Abschn. 8.3.3). 


\subsubsection{Analyse und Planung der Umsetzung}

Durch die Verwendung des InAsPro-Reifegradmodells im Softwaredemonstrator [37] wird der Digitalisierungsgrad von GRIMME für das Gesamtunternehmen bestimmt. Die vier zugehörigen Reifegradstufen sind 1) Erkunder, 2) Anfänger, 3) Fortgeschrittener und 4) Experte. Aufgrund eines aus dem Softwaredemonstrator resultierenden Digitalisierungsgrades von 3,0 zählt Grimme zu den fortgeschrittenen Unternehmen im Bereich Digitalisierung. Dies bedeutet, dass die Digitalisierung überwiegend in den Unternehmensbereichen umgesetzt wird. Dabei sind die Bereiche größtenteils horizontal und vertikal vernetzt. Weiterhin ist die Digitalisierung in der Unternehmenskultur verankert [29].

Der Digitalisierungsgrad von GRIMME setzt sich aus dem unternehmensübergreifenden Reifegrad und aus dem Reifegrad der Produktlebenszyklusphase Aftersales zusammen (Abb. 8.7, links). Weiterhin wird der Digitalisierungsgrad anhand der Dimensionen Technologie, Organisation, Mensch und Strategie aufgeschlüsselt (Abb. 8.7, rechts) und in Radardiagrammen visualisiert. Mithilfe dieser Radardiagramme können Stärken und Schwachstellen identifiziert werden. Grimme weist z. B. in der Dimension Organisation einen Digitalisierungsgrad von 2,9 auf und in der Dimension Technologie einen Digitalisierungsgrad von 3,3. Das Beispiel zeigt, dass im Bereich Organisation mehr Potenzial zur Digitalisierung möglich ist, als im Bereich der Technologie, welche sich als Stärke des Unternehmens herausstellt.

Das InAsPro-Reifegradmodell zeigt den aktuellen Digitalisierungsgrad des Unternehmens auf, jedoch wird kein Zielzustand definiert. Dieser wird im nächsten Schritt mithilfe der Digitalisierungsstrategien festgelegt.

Grundlage für die Ermittlung der Digitalisierungsstrategie ist die bereits vorhandene Unternehmensstrategie, die im Fall von GrImme eine Differenzierung des Produktportfolios anstrebt. Aufbauend darauf sowie auf dem ermittelten Digitalisierungsgrad

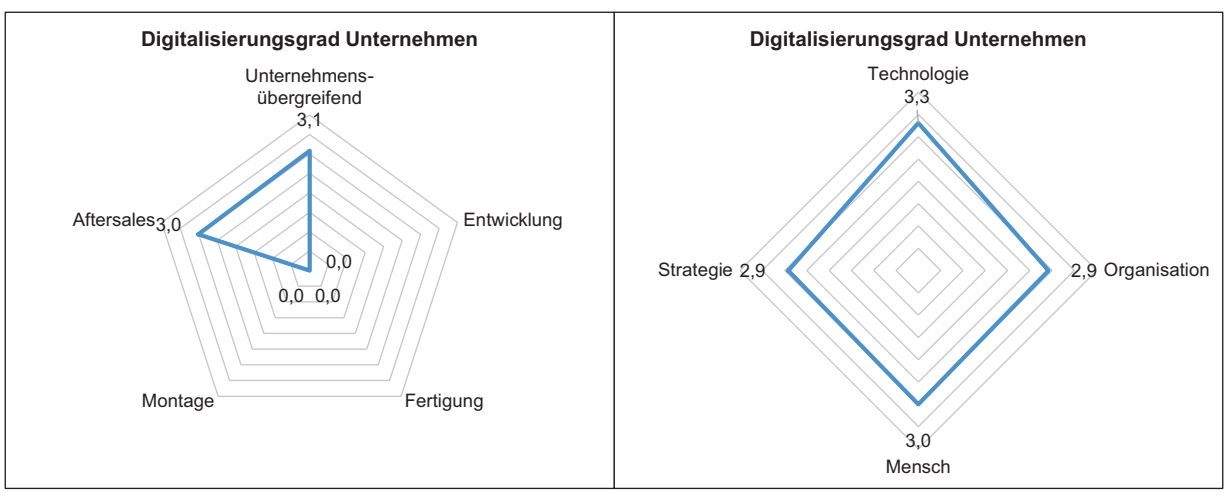

Abb. 8.7 Digitalisierungsgrad des Unternehmens Grimme 
für Grimme wird das strategische Handlungsfeld Prozesse und das damit verbundene strategische Ziel Prozesse automatisieren und vernetzen ausgewählt. Mithilfe der anschließenden Strengths-Weaknesses-Opportunities-Threats (SWOT)-Analyse werden im Softwaredemonstrator strategische Optionen abgeleitet [37]. Diese werden vom Unternehmen anhand der Kriterien Aufwand (Zeit für Schnittstellenprogrammierung und -definition, Zeit für Umsetzung) und Nutzen (Reduzierung der Zeit für Mehrfacheingaben, Kostenreduzierung bei der Einführung bei weiteren Partnern) sowie anhand der Auswirkungen auf die Dimensionen Mensch, Technologie und Organisation bewertet. Auf Grundlage der Visualisierung der bewerteten Optionen wählt GRIMME zwei im Rahmen der Pilotanwendung zu verfolgende strategischen Optionen aus: Die Standardisierung der Schulungsverwaltung im gesamten Unternehmen sowie auch bei Partnern. Zur Konkretisierung werden für beide strategischen Optionen messbare Zielwerte sowie notwendige Investitionen festgelegt. Messbare Zielwerte sind beispielsweise der erste Test mit sechs Nutzern des neuen IT-Systems nach ca. 20 Monaten Projektlaufzeit oder die Nutzung des neuen IT-Systems von $80 \%$ der Schulungsteilnehmer in den Ländern England und Frankreich bis in drei Jahren [6, 35].

Aufbauend auf der definierten Digitalisierungsstrategie wird die passende Digitalisierungslösung mithilfe des Technologieatlas im Softwaredemonstrator ausgewählt [36, 37]. Durch den Filterprozess wird das Anwendungsszenario „Unterstützung der Schulungsplanung“ konkretisiert. Mögliche Digitalisierungslösungen in diesem Anwendungsszenario sind das Kursverwaltungssystem sowie Onlineplanungs- und Qualifizierungstool. Für beide Lösungen erhält GRIMME Informationen zu benötigten Hard- und Softwarekomponenten, Chancen und Risiken für Mitarbeiter und Unternehmen sowie zu Wechselwirkungen mit anderen Digitalisierungslösungen. Letztlich wählte Grimme die Digitalisierungslösung Kursverwaltungssystem aus, das im Rahmen des Projekts InAsPro als Pilotanwendung im Aftersales implementiert wird [35].

Aufbauend auf den theoretischen Erkenntnissen und der Auswahl des Kursveraltungssystems als passende Digitalisierungslösung, wird anschließend die Pilotanwendung geplant. Hierbei werden Steckbriefe für die Pilotanwendung erstellt sowie eine Potenzialanalyse durchgeführt. Diese hat das Ziel, ein gemeinsames Verständnis des detaillierten Istzustands zu erhalten. Basierend darauf wird die Umsetzung geplant und potenzielle Risiken werden analysiert. Anschließend werden die Anforderungen an eine technische Lösung definiert.

\section{Beschreibung der Pilotanwendung in einem Steckbrief}

Zur Veranschaulichung der Rahmenbedingungen der Pilotanwendung wird ein Steckbrief verwendet (Abb. 8.8). Hierbei wird die Idee für die Pilotanwendung strukturiert erfasst und konkretisiert. Der Steckbrief enthält in Kurzform alle relevanten Informationen über die Pilotanwendung. Er legt eindeutig Inhalte, Ziele, Nutzen, Herausforderungen und Verantwortliche bzw. Beteiligte fest [18]. Für einen ersten Überblick wurde die Pilotanwendung kurz beschrieben und durch ein Schaubild visualisiert. Aufbauend auf der Einordnung in die Produktlebenszyklusphase Aftersales und die 


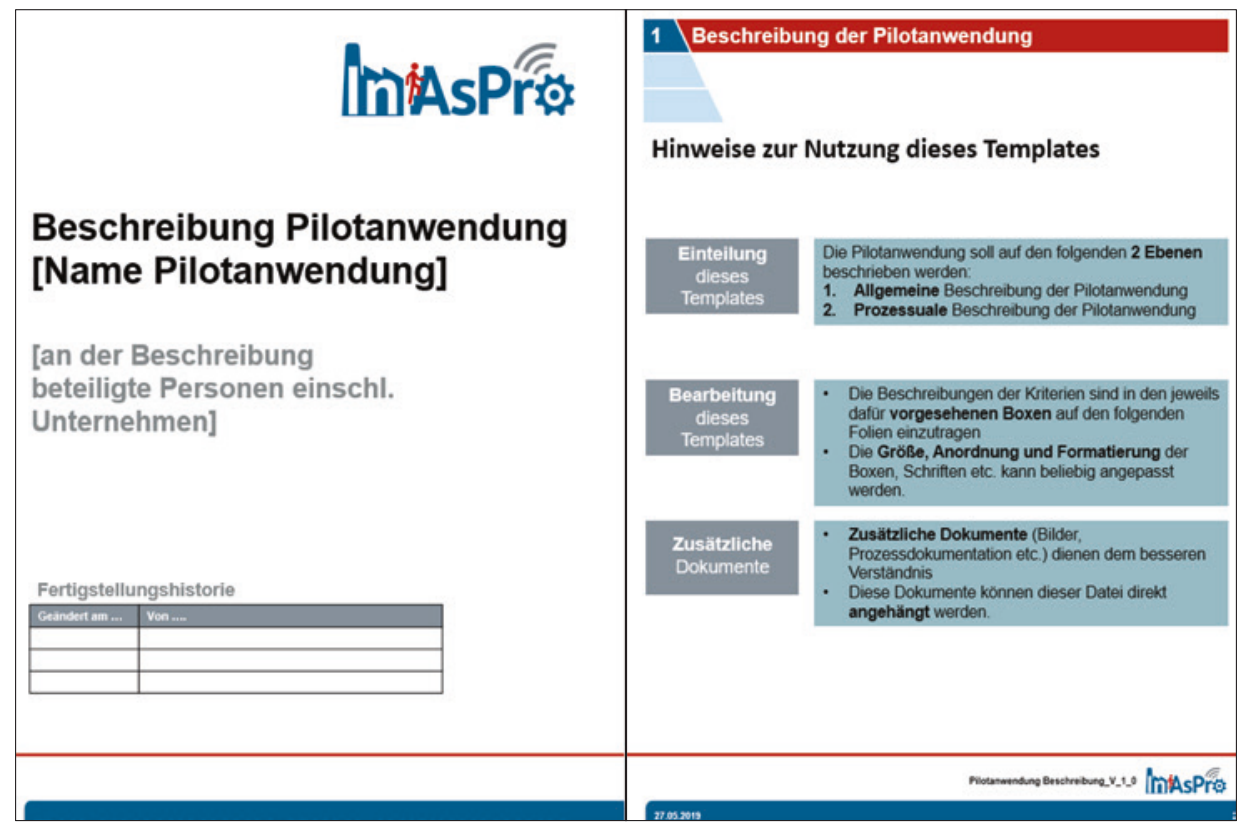

Abb. 8.8 Template Steckbrief für die Beschreibung der Pilotanwendungen

Dimensionen Mensch und Organisation, wird im nächsten Schritt die Zielsetzung näher erläutert und mögliche Problemstellungen und Herausforderungen bei der Umsetzung der Pilotanwendung werden beschrieben. Die Pilotanwendung wird anschließen anhand des für die Umsetzung ausgewählten Arbeitssystems analysiert (siehe auch Abschn. 8.2). Dies geschieht in drei Schritten für jede Kategorie des Arbeitssystems: 1) die aktuelle Ausgangssituation zum Projektstart, 2) der angestrebte Zielzustand des digitalisierten Arbeitssystems und - sofern bereits in Planung - 3) die Entwicklungsschritte, die hierfür notwendig sind, insbesondere die angestrebte Digitalisierungslösung. Mitunter sind nicht in allen Kategorien Veränderungen durch die Umsetzung der Pilotanwendung zu erwarten. Dies kann mithilfe der Kurzbeschreibung erkannt werden.

\section{Potenzialanalyse in der Pilotanwendung und Einbindung der Mitarbeiter in den Planungsprozess}

Um die Mitarbeiter frühzeitig in die Planung der Pilotanwendung einzubinden, werden Interviews bei GRIMME durchgeführt [31]. Neben Mitarbeitern werden auch Führungskräfte zur aktuellen Situation im Unternehmen sowie zu den geplanten Veränderungen befragt. Das Ziel ist es, eine detaillierte Analyse des Istzustands zu erhalten, indem Bedenken, Wünsche und Ideen der Mitarbeiter erfasst und den Einschätzungen der Führungskräfte gegenübergestellt werden. Aus den Befragungsergebnissen können anschließend Implikationen für die Umsetzung der Pilotanwendung extrahiert werden. 
Durch die Gegenüberstellung der Einschätzung von Mitarbeitern und Führungskräften können zudem Kommunikations- und Anpassungsbedarfe abgeleitet werden [35].

Im Rahmen der Interviews werden vier Bereiche adressiert: Technologie, Mitarbeiter, Digitalisierungsstrategie und Einführungsprozess. Im Bereich Technologie zeigen die Befragungsergebnisse, dass sowohl Mitarbeiter als auch Führungskräfte zu ähnlichen Einschätzungen hinsichtlich der technischen Ausstattung des Unternehmens kommen. Digitale Technologien werden bei GRIMME bereits in allen Unternehmensbereichen eingesetzt, wobei vielfältige Lösungen genutzt werden, z. B. das Grimme-ConneCT-System, das auch im Rahmen der Pilotanwendung als Grundlage der technischen Lösung fungiert. Die Weitergabe von Informationen über Systemschnittstellen hinweg und auch die Integration von Daten aus verschiedenen Systemlösungen in ein Gesamtsystem erfolgt im Unternehmen hingegen bisher nur teilweise automatisch. Dem möchte das Unternehmen durch die Pilotanwendung begegnen [35].

Das größte Potenzial der Pilotanwendung sehen Führungskräfte und Mitarbeiter dabei in der Steigerung der betrieblichen Effizienz und Kundenzufriedenheit. Auch der Arbeitsaufwand für Mitarbeiter soll sich deutlich reduzieren, da Prozesse vereinfacht und Fehler durch die digitale Informationsbereitstellung und -weitergabe vermieden werden können. Als größte Risiken bei der Umsetzung der Pilotanwendung wird vonseiten der Führungskräfte der Einführungszeitpunkt der technischen Lösung gesehen. Die Kursverwaltung ist stark saisonal geprägt, da die Kurstermine für das Folgejahr im Herbst bekanntgegeben werden und sich die Servicetechniker daraufhin für Schulungen anmelden. Daher sollte eine Einführung des KVS idealerweise im Sommer stattfinden. Die Mitarbeiter sehen neben dem Risiko des Systemausfalls auch die Möglichkeit, dass ineffiziente Arbeitsprozesse entstehen, falls nicht alle Mitarbeiter das neue System nutzen. So können parallele Arbeitsprozesse etabliert werden, die zu einem erhöhten Koordinationsaufwand führen würden.

Im Bereich Mitarbeiter werden die zuvor beschriebenen Elemente des Arbeitssystems sowohl in ihrem Ist- als auch in Ihrem Zielzustand betrachtet. Die bisherige Arbeitsaufgabe der Kursverwaltung wird von den Mitarbeitern meist als abwechslungsreich charakterisiert. Zwar sind die Aufgaben festgelegt, dennoch existiert eine zeitliche Flexibilität der Aufgabenbearbeitung. Zukünftig wird sich die Arbeitsaufgabe aus Sicht der Führungskräfte jedoch stark verändern, da die eigentliche Arbeitsaufgabe der manuellen Kursverwaltung entfällt und neue Tätigkeiten, beispielsweise die Ermittlung von Kompetenzbedarfen, entstehen. Dabei sehen Mitarbeiter und Führungskräfte gleichermaßen das Risiko, dass einzelne Mitarbeiter die neue Arbeitsaufgabe nicht akzeptieren oder überfordert sind. Dem soll frühzeitig mit entsprechenden Maßnahmen entgegengewirkt werden, wobei sowohl Führungskräfte als auch Mitarbeiter die Notwendigkeit sehen, Schulungen für alle Mitarbeiter anzubieten, um die neuen Arbeitsaufgaben und den Umgang mit dem KVS zu erlernen. Chancen für die Mitarbeiter entstehen, da der manuelle Arbeitsaufwand deutlich reduziert wird und Fehler vermieden werden können. Die so entstehenden zeitlichen Freiräume können für neue Arbeitsaufgaben genutzt werden, wodurch Entwicklungsmöglichkeiten geschaffen werden. 
Hinsichtlich des geplanten Einführungsprozesses zeigen die Interviews, dass sowohl aus Mitarbeiter-, als auch aus Führungskräftesicht noch Verbesserungsmöglichkeiten bei der Einbindung in die Pilotanwendung und die Kommunikation der Veränderungen bestehen. Um dem entgegenzuwirken, planen die Führungskräfte die Mitarbeiter an der Entwicklung der technischen Lösung zu beteiligen und Testrunden mit Key Usern durchzuführen. In diesen Testrunden kann das Feedback der Key User erfasst und anschließend eingearbeitet werden. Zudem sollen die Mitarbeiter regelmäßig in außerordentlichen Terminen und monatlichen Meetings über den aktuellen Umsetzungsstand der Pilotanwendung informiert werden. Die gesammelten Informationen der Interviews werden in der Umsetzungsplanung genutzt.

\section{Umsetzungsplanung der Pilotanwendung}

Für die Planung zur Umsetzung der Pilotanwendung werden drei Schritte durchgeführt. In einem ersten Schritt wird eine Grobplanung der Umsetzung basierend auf den zuvor durchgeführten Interviews erstellt, welche einen ersten Überblick über die Pilotanwendung gibt. In einem weiteren Schritt wird diese dann weiter konkretisiert und in ein Gantt-Chart überführt. Um die Zielerreichung der Umsetzung gewährleisten zu können wird im letzten Schritt eine Risikoanalyse durchgeführt. Die identifizierten Risiken werden bewertet und entsprechende Gegenmaßnahmen definiert [31].

Die Grobplanung basiert auf einem Veränderungsprozess nach [38], der vier Phasen unterscheidet: Orientierung, Planung, Realisierung und Anpassung. In der Orientierungsphase werden Trends und Entwicklungen im Umfeld des Unternehmens vor dem Hintergrund der Unternehmensstrategie analysiert, um mögliche Optionen für die Veränderung $\mathrm{zu}$ bestimmen. Aus diesen Überlegungen werden Ziele abgeleitet, die in der Planungsphase weiter konkretisiert werden. Dabei werden Anpassungsbedarfen im technologischen und organisatorischen Bereich und Mitarbeiterinteressen berücksichtigt. In der Realisierungsphase wird die Veränderung umgesetzt, bevor in der Anpassungsphase Verbesserungspotenziale ermittelt und umgesetzt werden. Basierend auf den Ergebnissen der Interviews wurde eine Grobplanung für GRIMME entwickelt, welche in Abb. 8.9 dargestellt ist.

Aufbauend auf der Grobplanung wird im Rahmen eines Workshops die Umsetzung weiter detailliert und in einem Gantt-Chart zusammengefasst (Abb. 8.10). Die Umsetzung erfolgt in vier Schritten: 1) Festlegen der Kern-Team Mitglieder, 2) Aufgaben mit Dauer festlegen und in Zeitplan einordnen, 3) Abhängigkeiten zwischen den Aufgaben identifizieren sowie 4) Identifikation des kritischen Pfades [20, 31].

Im ersten Schritt wird das Kern-Team festgelegt. Dieses besteht bei GRIMME intern aus einem Mitarbeiter im Aftersales, einem Mitarbeiter der Grimme Academy sowie aus Mitarbeitern aus der IT-Abteilung.

Im zweiten Schritt werden die notwendigen Aufgaben zur Umsetzung mit ihrer Dauer festgelegt und in einen Zeitplan eingeordnet. Zunächst werden die Aufgaben zur Umsetzung der Pilotanwendung gesammelt, wie z. B. die Planung der Ressourcen 


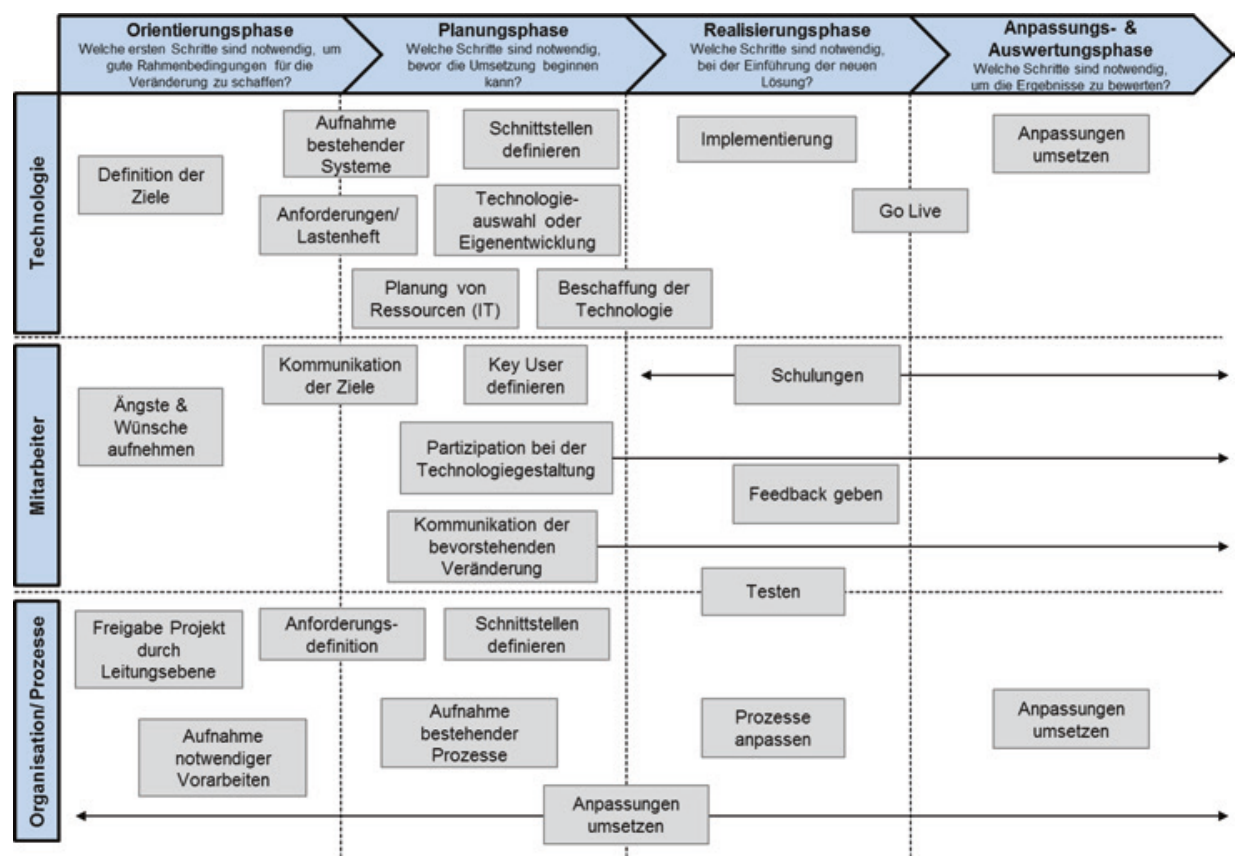

Abb. 8.9 Grobplanung der Pilotanwendung bei Grimme

in der IT, die Definition der Key User (intern und extern) oder auch die Durchführung von internen Schulungen für Key User während der Testphasen in der Entwicklung. Dabei werden mehrere Teilaufgaben zu einem Arbeitspaket (AP) zusammengeführt. Anschließend werden jeder Aufgabe eine Umsetzungsdauer sowie eine verantwortliche Person zugeordnet. Die genannte Person ist während der Umsetzung sowohl für die inhaltliche Bearbeitung der Aufgabe als auch für die Einhaltung des zugehörigen Zeitplans verantwortlich. Zur Erstellung des Zeitplans werden die identifizierten Aufgaben chronologisch geordnet und um fehlende Aufgaben ergänzt. Zusätzlich werden Meilensteine definiert, welche als Orientierungshilfen bei der Umsetzung dienen, da sie Zielzustände beschreiben, die bei der Umsetzung zu einem bestimmten Zeitpunkt erreicht werden müssen. Meist liegt zwischen den Meilensteinen ein relativ kurzer, zeitlicher Abstand, was das Überwachen des Fortschritts der Umsetzung erleichtert.

Im dritten Schritt werden die Abhängigkeiten zwischen den einzelnen Aufgaben identifiziert. Wenn ein Lastenheft entwickelt wurde und ein Projektteam aus der IT-Abteilung zur Umsetzung zur Verfügung steht, können erst Schnittstellen des einzuführenden IT-Systems hinsichtlich weiterer Technologien, der Organisation und vorhandener Prozesse definiert werden. Das IT-System wird externen Händlern erst nach dem GO LIVE, also der Inbetriebnahme des Systems unter realen Bedingungen, vorgestellt und somit auf dem Markt eingeführt. 


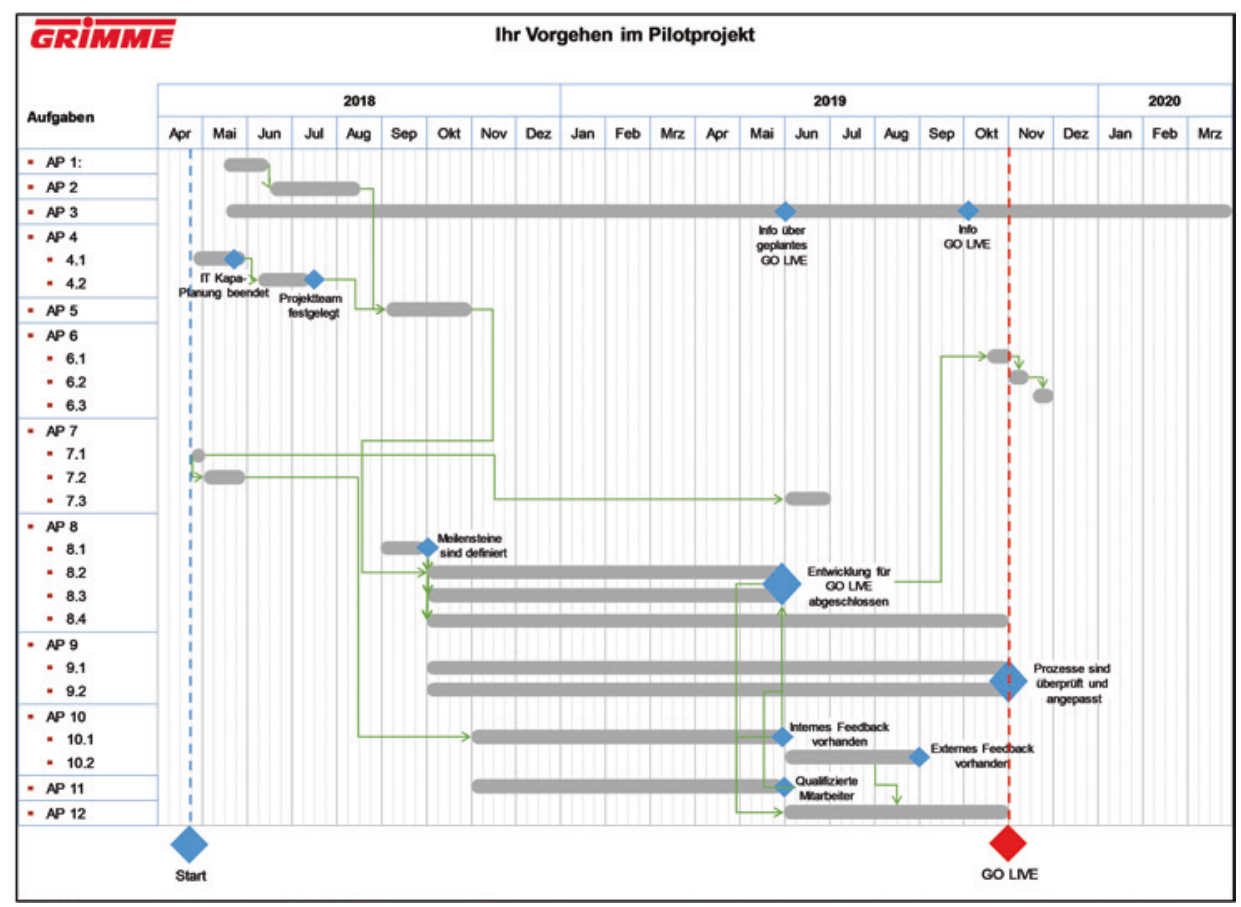

Abb. 8.10 Gantt-Chart der Pilotanwendung bei Grimme [35]

Im vierten Schritt wird der kritische Pfad anhand der zuvor identifizierten Abhängigkeiten bestimmt. Die längste Kette voneinander abhängenden Aufgaben stellen den kritischen Pfad dar. Verzögern sich Aufgaben auf dem kritischen Pfad, so verlängert sich das Gesamtprojekt, da die nachfolgenden Aufgaben nicht rechtzeitig begonnen werden können. Um dies zu vermeiden ist es wichtig, dass den Aufgaben auf dem kritischen Pfad eine hohe Priorität zugeordnet wird.

\section{Risikoanalyse}

Um potenzielle Risiken bei der Umsetzung der Pilotanwendung identifizieren zu können, wird eine Risikoanalyse anhand der Kategorien Markt, Technik, Termin, Kosten, Qualität und Mensch durchgeführt [20]. Für GrimmE wurden neun Risiken identifiziert: Ein Technik-Risiko ist bei Grimme z. B., dass das zu entwickelnde IT-System intern nicht anschlussfähig an andere Systeme ist oder zu langsam läuft. Weiterhin stellt die Entwicklerkapazität ein Termin-Risiko dar. Da im Unternehmen viele verschiedene Entwicklungsprojekte durchgeführt werden und dabei meistens die IT-Abteilung eingebunden ist, sind deren Kapazitäten eingeschränkt, was zu Verzögerungen führen kann. Weiterhin stellen notwendige Anpassungsmaßnahmen des IT-Systems auf Grundlage des Feedbacks der Key-User ein Termin-Risiko dar. Dadurch würde der Fortschritt im Projekt deutlich verzögert werden [31]. 
Die identifizierten Risiken werden anschließend bezüglich ihrer Auftretenswahrscheinlichkeit (P) und ihrer möglichen Auswirkungen (A) jeweils auf einer Skala von 1 (sehr gering) bis 5 (sehr hoch) bewertet. Je höher die Bewertung für A und P ist, desto wichtiger ist die Definition von Gegenmaßnahmen zur Vermeidung dieser Risikofaktoren [20]. Die Risiken 3 und 6 erhalten die höchste Bewertung hinsichtlich Auftretenswahrscheinlichkeit und Auswirkung (Abb. 8.11). Für beide werden entsprechende Gegenmaßnahmen zur Risikominimierung definiert.

\section{Anforderungsdefinition an die technische Lösung}

In einem nächsten Schritt werden anhand der bereits definierten Ziele Anforderungen an eine technische Lösung abgeleitet und in einem Anforderungskatalog gesammelt. Dadurch kann die Anzahl der potenziell einsetzbaren Digitalisierungstechnologien verkleinert werden [2]. Die Anforderungen werden in Muss- und Kann-Kriterien unterteilt. Muss-Kriterien sind Anforderungen, welche zwingend erfüllt sein müssen, damit die Digitalisierungslösung funktionsfähig ist und die gesetzten Ziele erreicht werden können. Kann-Kriterien sind optional Anforderungen. Auch ohne ihre Erfüllung ist die Inbetriebnahme der Digitalisierungslösung möglich [25]. Meist bilden Kann-Kriterien Zusatzfunktionen ab, die zwar einen höheren Nutzen generieren, jedoch häufig auch mit überproportional erhöhten Kosten verbunden sind. Ein Auszug des Anforderungskatalogs von Grimme für die Pilotanwendung ist in Tab. 8.1 dargestellt.

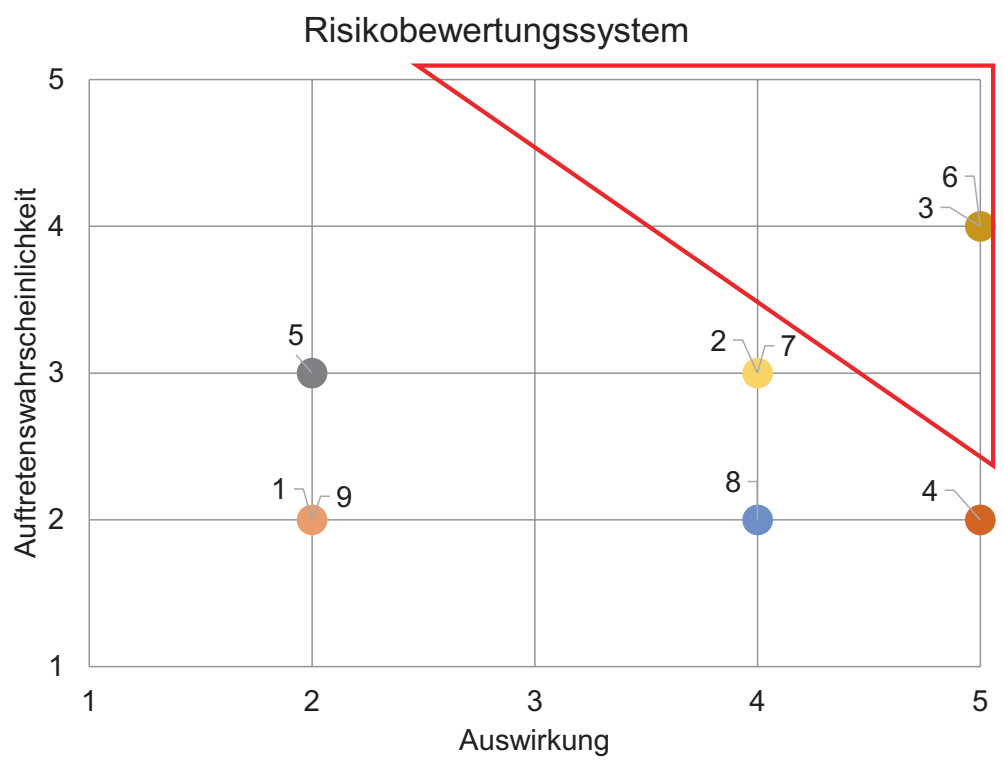

Abb. 8.11 Risikoanalyse der Pilotanwendung 
Tab. 8.1 Auszug aus dem Anforderungskatalog von GRIMME [35]

\begin{tabular}{|c|c|c|}
\hline Anforderungen & Muss-Kriterium & Kann-Kriterium \\
\hline \multicolumn{3}{|l|}{ Systemeinbindung } \\
\hline $\begin{array}{l}\text { Kompatibel mit GRIMME ConNECT } \\
\text { - Verknüpfung mit Schulungsinhalten } \\
\text { - Single Sign On (für Händler) }\end{array}$ & $\begin{array}{l}X \\
X\end{array}$ & \\
\hline $\begin{array}{l}\text { Verbindung mit MS Outlook } \\
\text { - Kalendereintrag zur Schulung für den Trainer } \\
\text { - Kalendereintrag für Teilnehmer }\end{array}$ & $\mathrm{X}$ & $\mathrm{X}$ \\
\hline \multicolumn{3}{|l|}{$\ldots$} \\
\hline \multicolumn{3}{|l|}{ Personal/Personaldaten } \\
\hline $\begin{array}{l}\text { Automatischer Abgleich Schulungshistorie + Schulungs- } \\
\text { anmeldungen } \\
\text { - Kursvoraussetzungen definieren }\end{array}$ & $\begin{array}{l}X \\
X\end{array}$ & \\
\hline $\begin{array}{l}\text { Schulungscoupons bei Anmeldung und Abrechnung berück- } \\
\text { sichtigen }\end{array}$ & & $X$ \\
\hline \multicolumn{3}{|l|}{$\ldots$} \\
\hline \multicolumn{3}{|l|}{ Verwaltung } \\
\hline $\begin{array}{l}\text { Automatischer Versand von Kursbestätigungen nach } \\
\text { Kontrolle/manueller Freigabe }\end{array}$ & $\mathrm{X}$ & \\
\hline Warteliste, wenn Kurs ausgebucht ist & & $\mathrm{X}$ \\
\hline \multicolumn{3}{|l|}{$\ldots$} \\
\hline \multicolumn{3}{|l|}{ Qualitätssicherung } \\
\hline Möglichkeit zum Feedback & $\mathrm{X}$ & \\
\hline Kursbezogene Gestaltung der Feedbackbögen & & $\mathrm{X}$ \\
\hline \multicolumn{3}{|l|}{$\ldots$} \\
\hline [Tabellenfußzeile - bitte überschreiben] & & \\
\hline
\end{tabular}

\subsubsection{Umsetzung bei GrIMME}

Ausgehend von der analysierte Pilotanwendung und der geplanten Umsetzung wird bei GRIMME ein Team zur Implementierung der Pilotanwendung aus dem Aftersalesbereich und der IT-Abteilung zusammengestellt. Dieses interdisziplinäre Team gliedert die Pilotanwendung zu Beginn in einzelne Arbeitspakete (Abb. 8.12) analog zum Gantt-Chart aus dem vorherigen Kapitel (vgl. Abschn. 8.3.1, Abb. 8.10). Die Abarbeitung der einzelnen Arbeitspakete erfolgt mithilfe agiler Methoden, u. a. mit Scrum. Dabei werden die Aufgaben in kurzen Sprints mit GRIMME-internen Softwareentwicklern umgesetzt.

In einem ersten Schritt zur Umsetzung der Pilotanwendung wurde der Istzustand nochmals genauer betrachtet und notwendige Vorabreiten zur Umsetzung der Pilotanwendung aufgenommen. Darauf aufbauend wurden die Anforderungen an ein 


\begin{tabular}{|c|c|c|c|c|}
\hline $\begin{array}{l}\text { Arbeits- } \\
\text { paket }\end{array}$ & \multicolumn{2}{|c|}{ Bezeichnung } & Verantwortliche Person & Lautzelt \\
\hline AP 1 & \multicolumn{2}{|c|}{ Aufnahme notwendiger Vorabreiten $\rightarrow$ „Blick uber den Tellerrand } & Hr. Stubbe & $15.05 .-15.06 .18$ \\
\hline AP 2 & \multicolumn{2}{|c|}{ Oberarbeitung Anforderungen / Lastenheft (Technologie + Prozesse) } & Hr. Stubbe & $15.06 .-15.08 .18$ \\
\hline AP 3 & \multicolumn{2}{|c|}{ RegelmäBige Kommunikation der Ziele (intern) } & Hr. Stubbe & $01.04 .18-30.03 .20$ \\
\hline \multirow[t]{3}{*}{ AP 4} & \multicolumn{2}{|c|}{ Ressourcenplanung IT } & Hr. Pier / Hr. Stubbe / Hr. Rahner & 23.04.-15.07.18 \\
\hline & AP 4.1 & Planung der Ressourcen in der IT & Hr. Rahner & $23.04 .-21.05 .18$ \\
\hline & AP 4.2 & Festlegen des Projektteams & Hr. Pier / Hr. Stubbe / Hr. Rahner & 15.06.-15.07.18 \\
\hline AP 5 & \multicolumn{2}{|c|}{ Schnittstellen definieren (Technologie + Orga / Prozesse) } & $\mathrm{Fr}$. Meyer / Hr. Ruf & 01.09.-01.11-18 \\
\hline \multirow[t]{4}{*}{ AP 6} & \multicolumn{2}{|c|}{ Kommunikation der bevorstehenden Ånderungen (extern) } & Fr. Meyer & $15.10 .-01.12 .19$ \\
\hline & AP 6.1 & Kommunikationsform festlegen (extern) & Fr. Meyer & $15.10 .-21.10 .19$ \\
\hline & AP 6.2 & Planung der benötigten Schulungen (extern) & Fr. Meyer / Hr. BlGmer & $22,10-11,11.19$ \\
\hline & AP 6.3 & Durchführung der Schulungen (extern) & Fr. Meyer & 12.11.-01.12-19 \\
\hline \multirow[t]{4}{*}{ AP 7} & \multicolumn{2}{|c|}{ Key User } & Hr. Stubbe / Fr. Meyer & 20.04.18-30.06.19 \\
\hline & AP 7.1 & Key User definieren (intern + extern) & Hr. Stubbe / Fr. Meyer & $20.04 .-30.04 .18$ \\
\hline & AP 7.2 & Absprache mit Key User (intern) & Hr. Stubbe / Fr. Meyer & 01.05.-31.05.18 \\
\hline & AP 7.3 & Absprache mit Key User (extern) & Fr. Meyer & 01.06.-30.06.19 \\
\hline \multirow[t]{5}{*}{ AP 8} & \multicolumn{2}{|c|}{ Entwicklung und Implementierung des Schulungsverwaltungssystems } & Fr. Meyer / Hr. Ruf & $01.09 .18-30.10 .19$ \\
\hline & AP 8.1 & $\begin{array}{l}\text { Festlegung der Meilensteine zur Entwicklung und Implementierung } \\
\text { (IT-seitig) }\end{array}$ & Fr. Meyer / Hr. Ruf & $01.09 .-30.09 .18$ \\
\hline & AP 8.2 & Durchführung Entwicklung und Implementierung & Hr. Ruf & $01.10 .18-31.05 .18$ \\
\hline & AP 8.3 & $\begin{array}{l}\text { Abnahme / Freigabe des entwickelten Systems (Zwischenstănde, GO LIVE-Stand; } \\
\text { Optimierungen) }\end{array}$ & Fr. Meyer / Hr. Ruf & $01.10 .18-31.05 .18$ \\
\hline & AP 8.4 & Testlaufe der entwickelten Technologie & Fr. Meyer / Hr. Ruf & $01.10 .18-31.10,18$ \\
\hline \multirow[t]{3}{*}{ AP 9} & \multicolumn{2}{|c|}{ Prozesse anpassen } & Hr. Stubbe & $01.09 .18-31.10,18$ \\
\hline & AP 9.1 & Profen der Prozesse auf Verănderung & Hr. Stubbe & $01,09.18-31,10.18$ \\
\hline & AP 9.2 & Prozesse nach Oberprüfung entsprechend anpassen (optional) & Hr. Stubbe & $01.09 .18-31.10 .18$ \\
\hline \multirow[t]{3}{*}{ AP 10} & \multicolumn{2}{|c|}{ Feedback } & Fr. Meyer / Hr. Ruf & $01.10,18-31.08 .18$ \\
\hline & AP 10.1 & Feedback der Key User (intern) & Fr. Meyer / Hr. Ruf & $01.10 .18-31.05 .18$ \\
\hline & AP 10.2 & Feedback der Key User (extern) & Fr. Meyer / Hr. Ruf & $01.06 .-31.08 .19$ \\
\hline AP 11 & \multicolumn{2}{|c|}{ Interne Schulungen fur Key User aber Testphasen in der Entwicklung } & Fr. Meyer & 01.10.18-31.05.19 \\
\hline AP 12 & \multicolumn{2}{|c|}{ Anpassungen der Entwicklung / Implementierung für GO LIVE } & Fr. Meyer / Hr. Ruf & 01.06.-31.10,19 \\
\hline
\end{tabular}

Abb. 8.12 Zeitplan zur Entwicklung der Pilotanwendungen bei Grimme

IT-System aus technologischer, aber auch aus organisatorischer Sicht analysiert und in einem Lastenheft festgehalten. Das Lastenheft präzisiert und ergänzt den Projektauftrag. Im Lastenheft werden alle Anforderungen beschrieben, die der Auftraggeber an die Erreichung des Projektziels stellt. Folgende Angaben werden typischerweise berücksichtigt: 1) Einführung, 2) Beschreibung des Ist-Zustands, 3) Beschreibung des Soll-Konzepts, 4) Beschreibung von Schnittstellen, 5) Funktionale Anforderungen, 6) Nichtfunktionale Anforderungen, 7) Risikoakzeptanz, 8) Skizze des Entwicklungszyklus und der Systemarchitektur oder auch ein Struktogramm, 9) Lieferumfang, 10) Abnahmekriterien [14].

Basierend auf dem Lastenheft wurden die notwendigen Ressourcen in der IT geplant. Ein wesentlicher Meilenstein im Projektfortschritt bei GrIMME ist die Planung und Freigabe der Ressourcen in der IT-Abteilung. Aufgrund der hohen Priorität der Digitalisierung bei Grimme stellen diese Ressourcen oft den Engpass bei der Umsetzung eines Projekts dar. Gleichzeitig wurde ein interdisziplinäres Team zur Umsetzung der Pilotanwendung zusammengestellt. Durch ein interdisziplinäres Team können verschiedene Anforderungen an das zu entwickelnde KVS berücksichtig werden. Weiterhin werden interne Key User bestimmt, die die interne Situation bei GRIMME gut beurteilen können. Ziel ist es, Arbeitserleichterung durch Digitalisierungstechnologien zu erreichen, indem möglichst viele manuelle Tätigkeiten digitalisiert werden. Zeitgleich 
muss aber abgewogen werden, welche Eigenschaften eine Hilfestellung bieten und welche eine Überbelastung durch zu viele Informationen zu Folge haben können. Durch die internen Key User erhielt das interdisziplinäre Team weitere Anforderungen und Verbesserungspotenziale für das KVS. Dadurch kann eine zielgerichtete Förderung durch die Anpassung an die Bedürfnisse des einzelnen Lernenden erreicht werden. So können die genutzten Medien z. B. angepasst werden an

- die jeweilige Arbeitssituation

- den Wissensstand

- den Qualifizierungsbedarf

- die Sprache [34].

Für weitere Tests werden externe Key User ausgewählt. Hierzu wählt GRIMME Mitarbeiter im Handel aus, die auch in der Vergangenheit schon bei der Entwicklung des Händlerportals Grimme unterstützt oder auch Erfahrung mit Systemen anderer Hersteller haben. Die ersten Tests mit externen Key Usern ergeben weitere Anforderung an die Nutzerfreundlichkeit eines Systems sowie auch Erkenntnisse zu notwendiger Information, die dem Servicetechniker bereitgestellt werden sollen. Diese werden bei der Entwicklung des Frontends berücksichtigt.

Zur informationstechnischen Umsetzung und Implementierung des KVS werden Meilensteine definiert. Bis zu diesen Zeitpunkten soll ein festgelegter Entwicklungsstand erreicht sein, welcher dann abgenommen und freigegeben wird. Nach Freigabe dieses jeweiligen Entwicklungsstandes können keine weiteren grundlegenden Änderungen vorgenommen werden, sodass nur eine Weiterentwicklung bzw. Optimierung des Zustandes des KVS möglich ist. Die finale Freigabe des KVS erfolgt für das GO LIVE.

Parallel zur Entwicklung werden Testläufe des KVS mithilfe der Key User durchgeführt und das erhaltene Feedback wird bei der Weiterentwicklung berücksichtigt. Diese Testphasen dienen ebenfalls dazu, die Key User in der Anwendung des KVS zu schulen. Demnach sind die Key User zum Zeitpunkt des GO LIVE in der Lage, das entwickelte KVS im Alltag anzuwenden.

Ebenfalls parallel zur Entwicklung des KVS werden zusammenhängende organisatorische Prozesse wie z. B. Workflows oder auch bestehende Arbeitsschritte auf mögliche Veränderungen geprüft. Um einen reibungslosen Einsatz des KVS gewährleisten zu können, werden diese Veränderungen nach der Überprüfung entsprechend angepasst.

Um eine flächendeckende Nutzung des KVS im Händlerportal GrimmE ConNECT zu realisieren muss dessen Verfügbarkeit an die Händler kommuniziert und gegebenenfalls Schulungen zu dessen Nutzung geplant werden. Anhand des Feedbacks der zuvor definierten Key User können die Schulungsbedarfe und -inhalte abgeleitet und bei der Durchführung der Schulungen berücksichtigt werden. Schulungen können hierbei vor Ort oder auch im Rahmen des Händlerportals mithilfe einer Schritt-für-Schritt-Anleitung durchgeführt werden. 
Während der Umsetzung der Pilotanwendung wird eine regelmäßige Kommunikation angestrebt, um so die Akzeptanz der Mitarbeiter gegenüber der neuen Technologie bzw. der Veränderung zu verbessern. Hierbei wird zu Beginn nicht nur die vom Management erarbeitete Vision an Mitarbeiter kommuniziert, sondern auch Erstinformationen zur anstehenden Pilotanwendung wie z. B. Name und Ziel der Pilotanwendung, strategischer Hintergrund, Meilensteine/Timing. Dabei ist die Informationstiefe von der Zielgruppe abhängig. Während der Realisierung der Pilotanwendung werden Erfolge offensiv kommuniziert. Nach der Umsetzung der Pilotanwendung werden die Erfolge konsolidiert und weitere Veränderungen eingeleitet. Weiterhin wird die Veränderungsbereitschaft in der Unternehmenskultur verankert [17, 22].

Zusätzlich zur Umsetzung der Pilotanwendung, wird das im Projekt InAsPro entwickelte Reifegradmodell angewandt (Abschn. 8.2) [29]. Anhand des resultierenden Digitalisierungsgrads können konkrete Handlungsbedarfe im Hinblick auf technologiegestützte Prozesse im Aftersales gewonnen werden. Basierend auf dem Gesamtergebnis und der entsprechenden Einstufungen hinsichtlich Digitalisierung können Tätigkeitsfelder der Abteilungen priorisiert und gezielt umgesetzt werden. Weiterhin können anhand des resultierenden Digitalisierungsgrads Informationen für die zu entwickelnde Digitalisierungsstrategie abgeleitet werden. Durch die Definition einer Digitalisierungsstrategie ergibt sich für den GrimME-Aftersalesbereich eine gute Möglichkeit, die aktuellen und noch geplanten Digitalisierungsvorhaben auf Umsetzbarkeit und Zielsetzung zu prüfen. Anhand der Bewertung können diese geplanten Digitalisierungsvorhaben priorisiert werden.

Erste Einblicke in das KVS sind in Abschn. 8.4.1 dargestellt. Das KVS wird aktuell im GRIMmE eigenen Händlerportal Grimme ConNECT weiter umgesetzt, mit dem Ziel diese ab Herbst 2019 zu nutzen.

\subsubsection{Unterstützende Methoden bei der Umsetzung}

Im Rahmen der Pilotanwendung wird zwischen den beteiligten Abteilungen und Akteure Regelmeeting initiiert und durchgeführt. Durch den Austausch mit allen Beteiligten sollen Herausforderungen und mögliche Risiken frühzeitig identifiziert und bewältigt bzw. minimiert werden, bevor daraus Probleme entstehen können, die die Umsetzung der Pilotanwendung verzögern oder gefährden könnten. Weiterhin stehen die Abteilungen für fachlichen Input zur Verfügung, indem sie aktuelle Erkenntnisse einbringen und somit Lösungsalternativen aufzeigen können.

Zur Unterstützung der Regelmeetings wird ein Template verwendet, welches den jeweiligen aktuellen Bearbeitungsstand widerspiegelt (Abb. 8.13). und so einen schnellen Überblick zur Pilotanwendung ermöglicht [1]. Der Stand der inhaltlichen Bearbeitung sowie die noch zur Verfügung stehende Zeit werden mithilfe einer Ampel angezeigt. Sind beide Ampeln grün, so erfolgt die Umsetzung der Pilotanwendung nach dem vordefinierten Zeitplan. Eine gelbe Ampel bedeutet eine inhaltliche bzw. zeitliche 


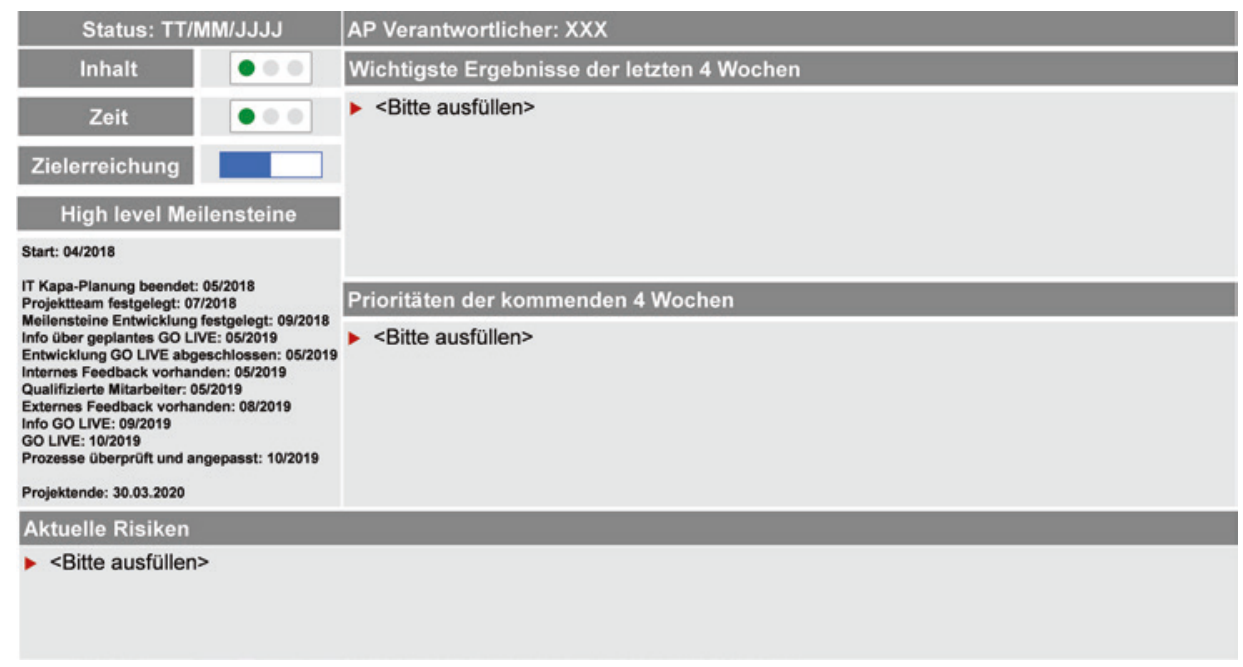

Abb. 8.13 Template der Regelmeetings

Verzögerung der Umsetzung. Hier sollten entsprechende Maßnahmen zur Problemlösung identifiziert und ungesetzt werden. Eine rote Ampel spiegelt einen Stillstand der inhaltlichen Bearbeitung bzw. zeitliche Probleme wider. Die Zielerreichung der Umsetzung wird durch eine Prozent-Leiste visualisiert. Je weiter die Pilotanwendung fortgeschritten ist, desto weiter nach rechts wird der Balken verschoben. Eine Orientierung hierfür liefert auch der Meilensteinplan. So können die wichtigsten Zielzustände immer im Blick behalten und bei möglichen Abweichungen Gegenmaßnahmen eingeleitet werden.

Neben den Statusanzeigen werden auch die inhaltlichen Aktivitäten genannt. Zum einen werden die wichtigsten Ergebnisse der vergangenen vier Wochen aufgezeigt und zum anderen die Prioritäten der kommenden vier Wochen. Für den folgenden Regeltermin vier Wochen später, werden die Inhalte neu eingefüllt. Im Idealfall werden die Aktivitäten aus den kommenden vier Woche zu den erledigten Aktivitäten der letzten vier Wochen. Ist dies nicht der Fall, wird geprüft wodurch die Verzögerung ausgelöst wurde und welche Maßnahmen ergriffen werden können, um den Zeitplan weiterhin einzuhalten. Zusätzlich werden identifizierte Risiken aufgeführt, für die gegebene Lösungsund Vermeidungsstrategien entwickelt werden.

\subsection{Ergebnisse}

Im Folgenden werden zunächst die erarbeiteten Ergebnisse der Pilotanwendung bei GRIMMEausführlich vorgestellt (Abschn. 8.4.1). Weiterhin werden die durch die Digitalisierung hervorgerufenen Auswirkungen auf die Arbeitsgestaltung (Abschn. 8.4.2) 
sowie auf die Produktivität (Abschn. 8.4.3) näher untersucht und erläutert. Das Kapitel schließt mit dem Beitrag der Ergebnisse der Pilotanwendung zu übergeordneten Zielen des Förderschwerpunkts (Abschn. 8.4.4).

\subsubsection{Darstellung der erarbeitenden Ergebnisse}

Bereits im Projekt InnoServPro [1] entwickelte GrIMME ein Kompetenzkonzept, um zukünftig PSS mithilfe von individualisierten, verfügbarkeitsorientierten Geschäftsmodellen anbieten zu können. Dabei steht das Zusammenwirken von Sach- und Serviceprodukten, auf der Grundlage von intelligent vernetzten Produkten und Serviceprozessen im Fokus der Betrachtungen. Hierfür wurden die technisch-organisatorischen Rahmenbedingungen auf Serviceseite (z. B. Kommunikationsanforderungen der Servicetechniker) in der Lebenszyklusphase des Aftersales betrachtet. Darauf aufbauend wurde in der Pilotanwendung in InAsPro ein IT-System entwickelt, mit dem Personalentwicklungsprozesse digital organisiert und auf die individuellen Bedarfe der Mitarbeiter abgestimmt werden können. Dabei wurde das IT-System an das bestehende Händlerportal (GRIMME CONNECT) angebunden.

Folgend werden nun die einzeln Tätigkeits- und Informationsschritte aufgezeigt, die im Rahmen einer Schulungsanmeldung von Aftersales-Mitarbeitern in Grimme ConNeCT durchzuführen sind [35]. Bisher wurden Schulungsanmeldungen rein papierbasiert verarbeitet. Zunächst wurde der Seminarkatalog ausschließlich in gedruckter Form an potenzielle Schulungsteilnehmer verschickt. Daraufhin erfolgte eine Anmeldung per Fax, woraufhin eine Kursbestätigung manuell erstellt und versendet wurde. Die Kursund Ressourcenplanung erfolgte in einem separaten Planungstool ohne Schnittstellen zu anderen Systemen. Auch hier wurde eine Integration angestrebt.Zur Zielerreichung wurde ein KVS entwickelt, welches den papierbasierten Seminarkatalog von GRIMME unmittelbar ersetzt (Abb. 8.14). Hier erhält der potenzielle Kursteilnehmer eine Übersicht, welche Qualifikationsmöglichkeiten angeboten werden, wobei neben dem Kursniveau auch das Potenzial des Kursangebots für den jeweilige Händler und die Service-Angebote aufgezeigt werden.

Um den Unternehmen eine einfache Möglichkeit zu bieten, Schulungsteilnehmer anzumelden und bspw. Reisezeiten und Unterbringung zu planen, sind zu den Kursangeboten jeweils alle Termine, Orte und Verfügbarkeiten abgebildet (Abb. 8.15). Wollten potenzielle Kursteilnehmer diese Informationen in der Vergangenheit ermitteln, waren häufig umfangreiche Recherche per Telefon und E-Mail erforderlich. Durch das neue KVS kann die Geschwindigkeit des Informationsaustauschs zwischen GRIMME und den Partnern erheblich gesteigert und eine Schulungsplanung im Moment der Informationsbereitstellung ermöglicht werden.

Auch über die Schulungsbuchung hinaus bietet das KVS den Partnern einen Mehrwert, indem sich der verantwortliche Mitarbeiter des Partners u. a. einen Gesamtüber- 


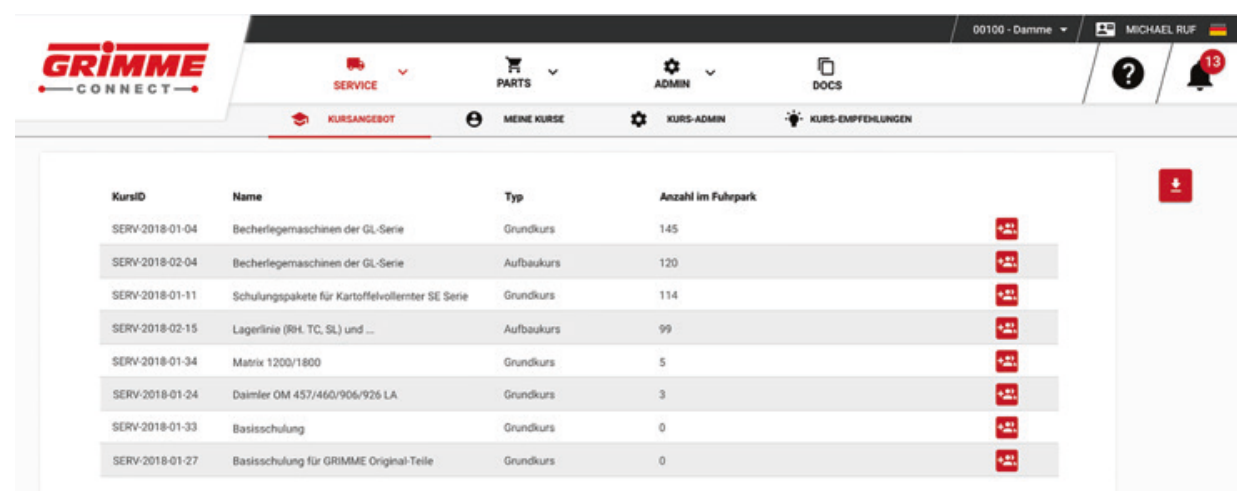

O GRiMME Landmaschinentabrik CembiH \& Ca. KG 2018

FAO

Abb. 8.14 Kursübersicht der verfügbaren Angebote

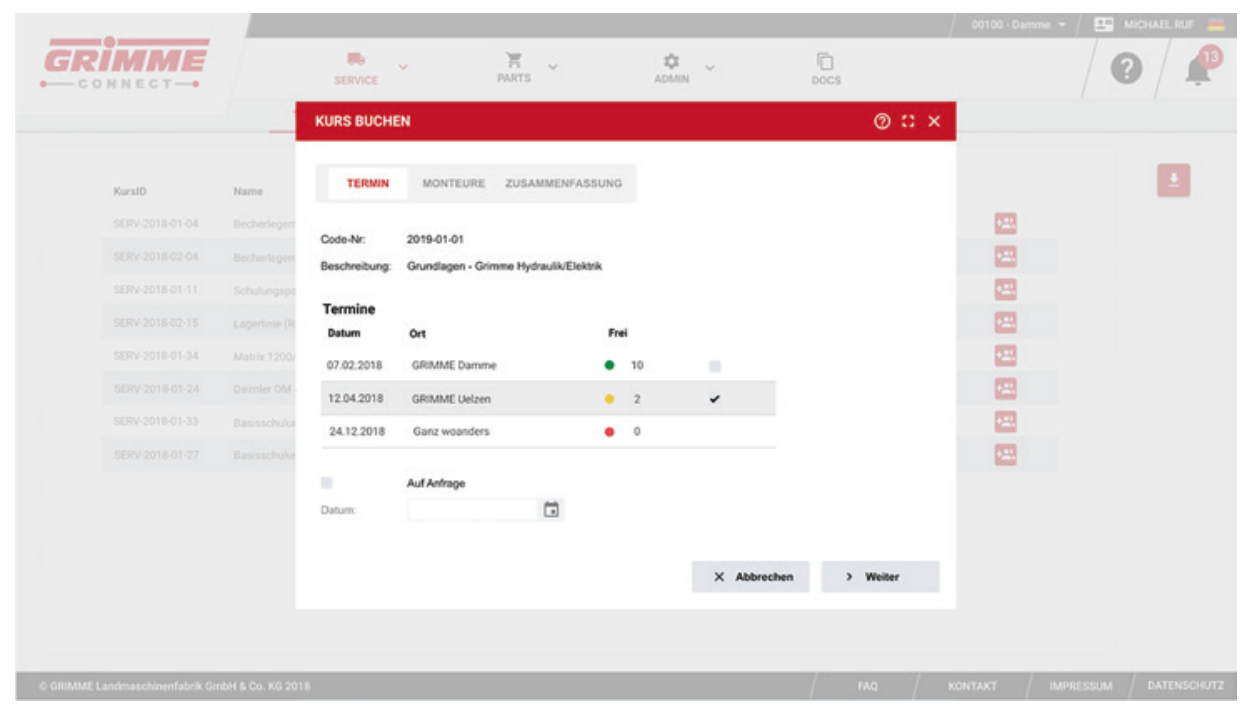

Abb. 8.15 Verfügbare Plätze mit Ort der Durchführung

blick über die Anzahl der besuchten Schulungen je Monteur und Jahr verschaffen kann (Abb. 8.16). Um Kunden einen Anreiz zu bieten, an den Schulungen teilzunehmen, hat Grimme ein Vergütungssystem etabliert. Es ermöglicht dem Partner bei der Abrechnung 


\section{DETAILS FÜR KARTOFFELVOLLERNTER SE 260 (TYP.04110)}

FUHRPARK MONTEURE KURSE

Fähigkeiten je Monteur im Zeitlichen Verlauf

\begin{tabular}{l|rrr|rrr} 
NAME & 2013 & 2014 & 2015 & 2016 & 2018 & Gesamt \\
\hline Herr Beuke, Morten & & & & 1 & 1 & $\mathbf{2}$ \\
Herr Kröger, Marek & & & & & 1 & $\mathbf{1}$ \\
Herr Martens, Cord & 1 & 1 & 1 & 1 & & $\mathbf{4}$ \\
\hline Herr Meyer, Alfred & 1 & 1 & 1 & 1 & 1 & $\mathbf{5}$ \\
Herr Schiller, Torsten & 1 & 1 & 1 & 1 & 1 & $\mathbf{5}$ \\
\hline Herr Spreen, Dieter & 1 & 1 & 1 & 1 & 1 & $\mathbf{5}$ \\
\hline Gesamt & $\mathbf{4}$ & $\mathbf{4}$ & $\mathbf{4}$ & $\mathbf{5}$ & $\mathbf{5}$ & $\mathbf{2 2}$
\end{tabular}

Abb. 8.16 Schulungshistorie als Gesamtübersicht pro Händler

seiner Arbeitseinsätze den Stundensatz um eine Stufe zu erhöhen, wenn mindestens ein Mitarbeiter des Partners eine entsprechende Schulung besucht hat. Dadurch profitiert der Partner von höheren Einkünften, während für GRIMME die Wahrscheinlichkeit steigt, dass Monteure an Schulungen teilnehmen.

Eine weitere Ansicht im Kursverwaltungssystem zeigt die Fähigkeiten je Monteur über mehrere Jahre an (Abb. 8.17). Häufig beruht die Grundqualifikation der Monteure auf einer Berufsausbildung zum Land- und Baumaschinemechatroniker. Aufgrund der Bedarfe des Unternehmens oder den Entwicklungspotenzialen des Monteurs kann der Partner einen Qualifikationsaufbau planen. Mit Hilfe des KVS wird ersichtlich, welche Fähigkeiten der Monteur bereits erworben hat und ob eine Spezialisierung vorliegt.

Weiterhin kann der Händler im KVS seine ihm zugewiesen Maschinen identifizieren und nach Maschinentyp sortieren (Abb. 8.18). Darin erkennt er dann im zeitlichen Verlauf die Anzahl des ausgewählten Maschinentyps. Diese Übersicht kann er mit den Fähigkeiten seiner Mitarbeiter abgleichen und ggf. Weiterbildungsbedarfe in einzelnen Technologiebereichen identifizieren. Aufgrund der zusätzlichen Kompetenz können Servicetätigkeiten übernommen werden die in der Vergangenheit nur vom Hersteller selbst oder besonders qualifizierter Mitarbeiter durchgeführt werden durften. Darüber hinaus bildet der Partner durch seine Mitarbeiter den hohen Qualitätsanspruch von GRImme ab wofür der Kunde auch bereit ist einen höheren Stundesatz zu zahlen. Als dritte Säule reduziert ein Kompetenter Servicemitarbeiter durch schnelle Arbeitserledigung sowohl in Diagnose wie auch in der Reparatur die Stillstands Zeiten der Produkte beim Anwender. Somit kann der Partner mehr Service-Geschäft realisieren. Weiterhin hat der Partner durch die Systemanbindung an das Händlerportal GrimmE 


\section{DETAILS FÜR MONTEUR "Jasper van dem Berg"}

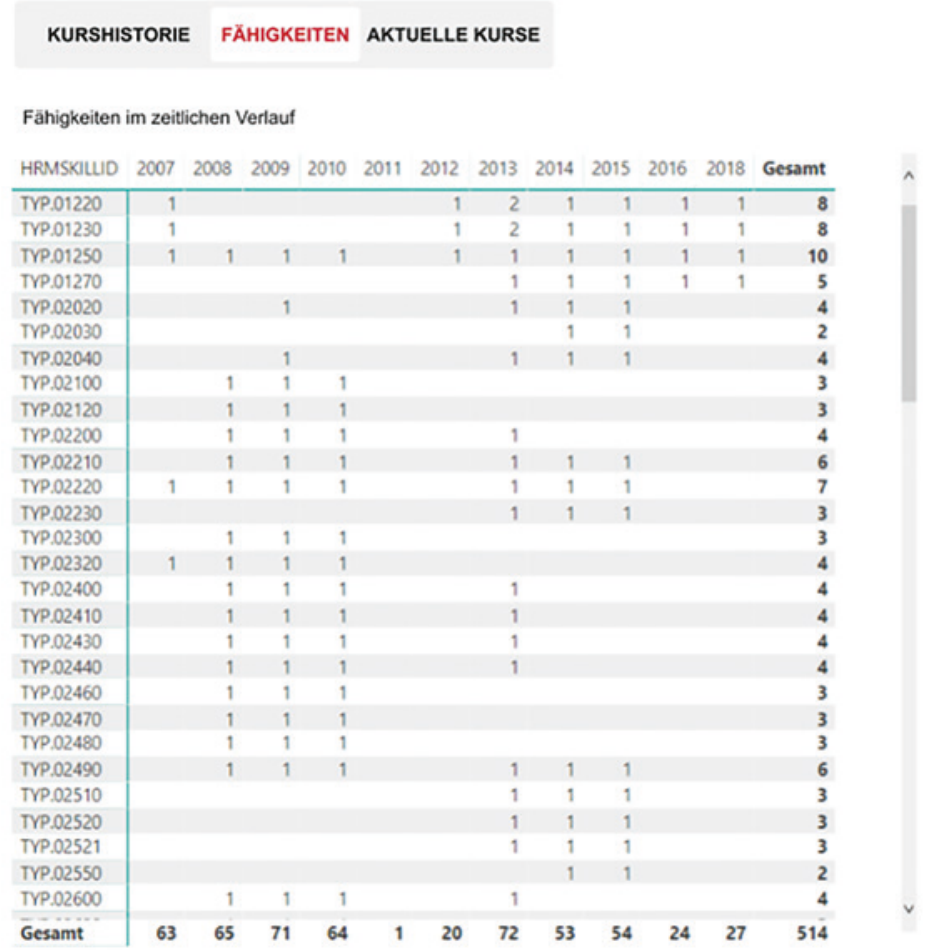

Abb. 8.17 Absolvierte Schulungen pro Mitarbeiter

Connect die Möglichkeiten sich einen Überblick über ausstehende Produktlieferungen zu verschaffen. Basierend auf den bestellten Produkten lassen sich ggf. zukünftige Weiterbildungsbedarfe der Mitarbeiter identifizieren.

\subsubsection{Auswirkungen auf die Arbeitsgestaltung}

Die beschriebenen Veränderungen bieten für GRIMME den Vorteil, dass Prozesse effizienter gestaltet werden und so nicht nur der Service für Kunden, sondern auch die Datenbereitstellung und -verarbeitung verbessert werden können. Gleichzeitig bietet die Umsetzung der Pilotanwendung das Potenzial, Aftersales-Mitarbeiter bei der Ausführung ihrer Arbeitsaufgabe zu entlasten. Papierbasierte, koordinations- und abstimmungsintensive Prozesse werden digitalisiert, wodurch Arbeitsschritte vereinfacht werden. Dadurch können Mitarbeiter die anfallenden Arbeitsaufgaben nicht nur in kürzerer Zeit erfüllen und somit Freiräume für neue Aufgaben erhalten. Auch Fehler werden ver- 


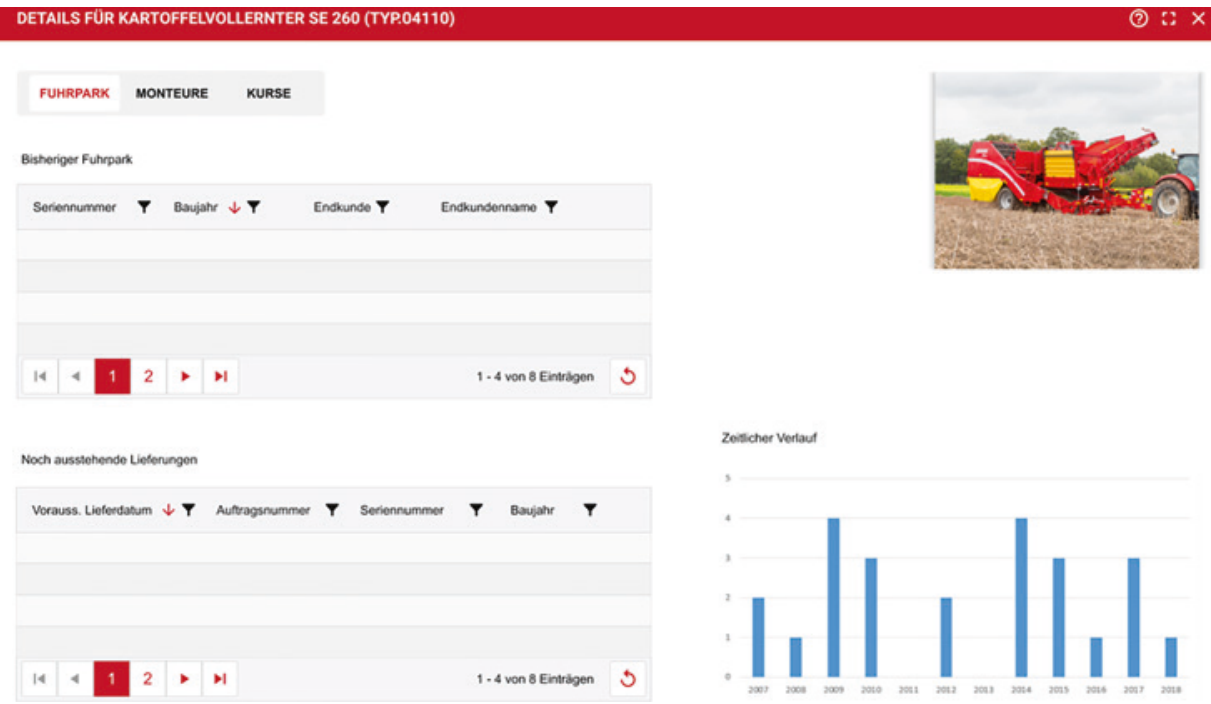

Abb. 8.18 Übersicht über Maschinen im Kundenstamm sowie folgende Maschinen

mieden, die früher durch das händische Übertragen von Informationen gemacht wurden. Durch die Digitalisierung des Arbeitsprozesses entstehen jedoch auch Risiken, denen GRIMME bei der Einführung des KVS begegnen musste. Übernimmt das IT-System zu viele der Arbeitsaufgaben von den Mitarbeitern, droht die Gefahr der Unterforderung und Monotonie. Die Arbeitsbelastung kann durch verringerte körperliche oder geistige Anforderungen so weit sinken, dass der mangelnde Trainingsreiz zu Abbau der Funktions- und Leistungsfähigkeit führen kann. Unterforderung kann dabei sowohl durch einen zu geringen Anspruch an die Qualifikationen des Mitarbeiters ausgelöst werden, als auch durch ein zu geringes Arbeitsvolumen [13]. Dem begegnet GRIMME dadurch, dass die Mitarbeiter weitere Aufgaben im Unternehmen übernehmen und so ihr Aufgabenspektrum erweitern.

Da das IT-System eine Vielzahl von Entscheidungen und Prozessen selbst ausführt, besteht die Gefahr, dass Mitarbeiter langfristig eine Technikabhängigkeit entwickeln. Dies geschieht, wenn die Interaktion von Menschen und Technik zu stark vertieft wird, sodass Arbeitsaufgaben ohne technische Unterstützung nicht mehr ausgeführt werden können [16]. Insbesondere für GRIMME stellt dies eine große Herausforderung dar. Die Firma fertigt und vertreibt komplexe, innovative technische Produkte, die von den Mitarbeitern ein ausgeprägtes technisches Verständnis, Analysefähigkeit sowie das Denken in vernetzten Prozessen fordern. Kontinuierliche Weiterbildungsangebote und ein intensiver Erfahrungsaustausch sind daher für das Unternehmen zwingende Voraussetzungen, um Wissen im Unternehmen zu halten und notwendige Kompetenzen weiter auszubauen. 


\subsubsection{Produktivitätswirkung bzw. Wirtschaftlichkeitsbetrachtung}

Durch die Einführung des KVS kann GrimmE die Wettbewerbsfähigkeit im Aftersales deutlich steigern und die derzeitige Marktposition stärken. Die im Projekt gewonnenen Erkenntnisse wird Grimme im Aftersales des Unternehmens implementieren. Somit werden nach Umsetzung der Pilotanwendung die Kompetenzen der Mitarbeiter im Aftersales bezüglich Digitalisierung gestärkt und stetig ausgebaut. Durch das Einsetzen einer Qualifikationsmatrix kann für jeden Mitarbeiter ein individuelles Kompetenzkonzept erstellt werden.

Aufgrund der Produktivitätseinschätzung unterschiedlicher Mitarbeiter (Alter und Nutzung der Systeme) wird die Digitalisierungslösung der Kursverwaltung bei GRIMME und GRIMME Partnern rund $20 \%$ Arbeitszeit in der Administration einsparen da es nun keine Systembrüche mehr durch papierunterstützte Arbeitsabläufe in der Kursauswahl gibt. Dies schließt auch einen positiven Produktivitätseffekt durch häufige Nutzung der Digitalisierungslösung durch die Prozess Innovation ein. Eine weitere Produktivitätswirkung kann sich in der GRIMME Gruppe durch Implementierung der Digitalisierungslösung im weltweiten Aftersalesnetzwerk bei Tochterunternehmen ergeben.

Im Rahmen der Produktivitätsbetrachtung wird es zunehmend wichtiger, nicht nur die Kommunikation mit Kunden zu verbessern, sondern auch einen reibungslosen Ablauf des Service beim Kunden sicherzustellen. Im besten Fall erreicht GrimmE eine Fehlerbehebung beim Kunden beim ersten Vor-Ort-Termin (sog. „First Visit Succes“). Hierfür ist eine professionelle Durchführung des Service mit detaillierter Planung und Einsatzorganisation, ein definierter Service-Prozess mit ausreichenden Standards, ein zeitnahes Controlling der Leistungserbringung und eine ausreichende Nähe zum Kunden unverzichtbar [24].

\subsubsection{Beitrag zu übergeordneten Zielen im Förderschwerpunkt}

Durch die angestoßene anwenderorientierte Transformation der Aftersales-Prozesse soll die Digitalisierung zusammen mit den Mitarbeitern bestmöglich gestaltet werden. Die Maßnahmen sollen dazu beitragen das übergeordnete wirtschaftliche Verwertungsziel die Verbesserung der Unternehmensprozesse - bei GRIMME erreicht wird.

Durch die Umsetzung der Pilotanwendung sammelt Grimme wichtige Ergebnisse, die in weiteren internen Projekten in allen Produktlebenszyklusphasen von Bedeutung sein werden. Zudem wird aufgrund des gewonnen Erfahrungswissens ein Abschätzen der Potenziale und Risiken der Digitalisierung sowie die Erfolgsaussichten der Realisierung in unterschiedlichen Arbeitssystemen hinsichtlich Mensch,Technik und Organisation für GRIMME erleichtert.

Durch die Entwicklung eines Transformationskonzepts sollen humane und organisatorische Arbeitsbedingen geschaffen werden die zu einer positiven Produktivi- 
tätswirkung führen wie im vorherigen Kapitel beschrieben. Die Ziele aus dem Projekt „Innovationen für die Produktion, Dienstleistung und Arbeit von morgen“ werden auch unternehmensintern fokussiert. Digitalisierung kann Arbeit substituieren, nur ist es bei dieser Lösung gelungen durch sozialverträgliche Arbeitsgestaltung und ergonomische Arbeitsbedingungen die Produktivität zu steigern und schon in der Planung der Lösung sehr praxisnah zu bleiben um die Umsetzung sicher zu stellen.

Ein Transfer dieser wissenschaftlichen und praktischen Ergebnisse des Projekts und der Pilotanwendung in die Praxis, erfolgt zum einen im eigenen GRIMME-Aftersalesbereich aber auch in den internationalen Tochterunternehmen. Die Verbreiterung der Projekterkenntnisse wird zudem durch die Mitgliedschaft in Arbeitskreisen, wie z. B. im Verband-Deutscher-Maschinen-und-Anlagenbau (VDMA)-Arbeitskreis „Kundendienst“ [5], sichergestellt. Somit können mittelfristig auch andere Unternehmen von den Ergebnissen des Projekts InAsPro profitieren.

\subsection{Lessons learned}

Im Rahmen der Analyse des Istzustands im Arbeitssystem mittels einer Potenzialanalyse können Stärken und Schwächen aufgedeckt werden. Anhand der Gegenüberstellung der Einschätzung der Mitarbeiter und der Führungskräfte wurden Kommunikations- und Anpassungsbedarfe abgeleitet. Die Planung der Umsetzung mittels eines Gantt-Charts ermöglichte das Sichtbarwerden der Aufgaben sowie ihrer Dauer zur Abarbeitung. Weiterhin wurden Zusammenhänge zwischen den Aufgaben visualisiert, sodass deutlich wurde, welche Aufgaben zuerst bearbeitet werden müssen, bevor eine andere begonnen werden kann. Zusätzlich kann durch die bildliche Darstellung der Arbeitsfortschritt dargestellt werden, sodass jeder Beteiligte zu jedem Zeitpunkt einen Orientierungspunkt hat. Dies dient gleichzeitig einem Soll-Ist-Vergleich. Die Durchführung einer Risikoanalyse ermöglichte den Mitarbeitern, sich mit potenziellen Risikosituationen im Projekt auseinander $\mathrm{zu}$ setzen, $\mathrm{zu}$ bewerten und geeignete Gegenmaßnahmen $\mathrm{zu}$ formulieren. Dadurch konnten die potenziellen Risikosituationen und ihre Bewertung dokumentiert und transparent dargestellt werden. Durch eine Priorisierung der Risiken konnte bereits bei kleinen Anzeichen proaktiv gegengesteuert und dadurch unnötige Aufwände wie z. B. Zeit oder Kosten vermieden werden.

Zur Umsetzung der Pilotanwendung wurde ein interdisziplinäres Team gebildet, in dem ebenfalls Mitarbeiter vertreten waren, welche später die gewählte Digitalisierungstechnologie anwenden. Dadurch konnten die Mitarbeiter ihre konkreten Mitarbeiterwünsche an die Digitalisierungstechnologie direkt miteinbringen und Experten aus den jeweiligen Abteilungen konnten zur Umsetzung das relevante Wissen beisteuern. Somit konnten die Mitarbeiter aktiv involviert und motiviert werden. Bei der Umsetzung der Pilotanwendung hat sich eine transparente, regelmäßige Kommunikation bei GRIMME bewährt. Die Regelmeetings wurden im zweiwochen-Rhythmus mit allen beteiligten Abteilungen und Akteuren durchgeführt, sodass die Abarbeitung der einzelnen Arbeits- 
pakete agil erfolgte. Dies ermöglichte den Beteiligten einen direkten Einfluss auf den Projektverlauf und führte zu effektiveren Arbeitsabläufen und schnelleren Ergebnissen. Weiterhin konnte bei Problemstellungen während der Umsetzung der Pilotanwendung flexibel und schnell reagiert werden.

\subsection{Zusammenfassung und Ausblick}

Durch die Globalisierung hat der deutsche Maschinenbau mehr als eine Milliarde Maschinen weltweit verkauft. Digitale Komponenten sowie eine digitale Infrastruktur ermöglichen eine globale Vernetzung dieser Maschinen, sodass sie weltweit von jedem Ort aus betrieben werden können [19]. Damit wird das traditionelle Wissen um Handel und Technik (Mechanik) infrage gestellt. Allerdings stellt dies gleichzeitig eine Chance für den Maschinenbau dar. Die Anforderungen der Digitalisierung können bereits in der Produktentwicklung berücksichtigt werden, sodass produzierte Maschinen mit digitalen Komponenten ausgestattet und mit digitaler Infrastruktur verknüpft werden. Dadurch bleibt der deutsche Maschinenbau wettbewerbsfähig und kann den Wirtschaftsstandort Deutschland nachhaltig sichern REF_Ref15649822 \n \h [3].

Die Digitalisierung wird in vielen Bereichen Wettbewerbsvorteile generieren. Sie wird aber auch die Beschäftigten vor die Herausforderung stellen, mit den verwendeten Digitalisierungstechnologien umzugehen. Dazu müssen die Mitarbeiter keine IT-Spezialisierung durchlaufen, sie sollten aber die grundlegenden Funktionen der Digitalisierungstechnologien kennen und den Umgang mit diesen beherrschen. GRIMME wird Weiterbildungsangebote für den Umgang mit Digitalisierungstechnologien schaffen, die die Mitarbeiter aktiv und auch selbstverantwortlich wahrnehmen können. Die Informationsweitergabe und Begründung, warum und wie eine neue Digitalisierungstechnologie eingeführt wird, kann schon zur Kompetenzentwicklung der Mitarbeiter beitragen. Die transparente Kommunikation dient aber auch dazu, Ängste und Befürchtungen abzubauen und dadurch die Motivation und die Begeisterung der Mitarbeiter für die Digitalisierung zu steigern. Zur Unterstützung des Angebots im Umgang mit Digitalisierungstechnologien bedarf es eines Kompetenzkonzeptes, basierend auf dem Digitalisierungsgrad des Unternehmens. Wird dieser nicht berücksichtigt, droht eine unzureichende Vorbereitung auf die Digitalisierung, da die Wissensvermittlung und der Kompetenzaufbau am Digitalisierungsgrad ansetzen. Erfahrungswissen und Intuition sind Bestandteile der Kompetenzen und Erfahrungswissen erfolgt aus Beschäftigung mit diesen Themen [33].

Das Wissen über den Digitalisierungsgrad des Unternehmens oder der betroffenen Arbeitsbereiche ist die Voraussetzung zur Innovation durch Digitalisierung. Es kann sonst passieren, dass eine Digitalisierungslösung gewählt wird die aufgrund der Mitarbeiter, Kompetenzen oder bereits vorhandener Technologien gar nicht umsetzbar ist. Dieser Digitalisierungsgrad wird vor der Auswahl der Technologie ermittelt und führt damit zum „Lernenden Unternehmen“ von Prozessinnovation durch Digitalisierung. 
Durch den im Projekt InAsPro entwickelten Technologieatlas erhalten unternehmensinterne Führungskräfte eine Orientierungshilfe bei der Prüfung verschiedener Digitalisierungslösungen. Die Beschreibung der Vor- und Nachteile auf Unternehmensund Mitarbeiterebene unterstützt dabei die Auswahl geeigneter Digitalisierungslösungen und zeigt Potenziale und Risiken auf [30,36]. GRIMme strebt eine Verankerung des Technologieatlas für weitere Verwendung und Nutzung im Unternehmen über das Projektende hinaus an.

Das entwickelte Transformationskonzept begleitet den Einführungsprozess von Digitalisierungstechnologien in produzierenden Unternehmen. Bei sorgfältiger Nutzung des ausgearbeiteten Transformationskonzeptes unter Berücksichtigung der betroffenen Mitarbeiter können Fehlentscheidungen frühzeitig vermieden werden und Digitalisierungstechnologien erfolgreich eingeführt werden [27].

\section{Literatur}

1. Aurich JC, Koch W, Kölsch P, Herder CF (2019) Entwicklung datenbasierter Produkt-Service Systeme. Ein Ansatz zur Realisierung verfügbarkeitsorientierter Geschäftsmodelle. Springer Vieweg

2. Balzert H (2009) Lehrbuch der Softwaretechnik: Basiskonzepte und Requirements Engineering. Spektrum Akademischer Verlag, Heidelberg

3. BMEL - Bundesministerium für Ernährung und Landwirtschaft (2018) Digitalisierung in der Landwirtschaft. Chancen nutzen - Risiken minimieren. https://www.bmel.de/SharedDocs/ Downloads/Broschueren/DigitalpolitikLandwirtschaft.pdf?__blob=publicationFile. Zugegriffen: 2. August 2019

4. Broy M (2010) Cyber-Physical Systems. Springer Berlin Heidelberg, Berlin, Heidelberg

5. Buschle P, Thomin P (2019) Arbeitskreis Kundendienst. https://www.vdma.org/v2viewer/-/ v2article/render/15963925. Zugegriffen: 2. August 2019

6. Dupont S, Siedler C, Tafvizi Zavareh M, Göbel JC, Zink KJ (2019) Entwicklung eines modularen und partizipativen Transformationskonzepts zur Digitalisierung produzierender Unternehmen. In: Gesellschaft für Arbeitswissenschaft e.V. Arbeit interdisziplinär. analysieren - bewerten - gestalten. GfA-Press. Dortmund

7. Dupont S, Siedler C. Tafvizi Zavareh, Schröder D (2020) Technologieatlas zur Auswahl von Digitalisierungslösungen. In: Aurich JC, Pier M, Siedler C, Sinnwell C (Hrsg) Bedarfsgerechte Digitalisierung von Produktionsunternehmen. Ein modulares Transformationskonzept als praxisorientierter Ansatz. Synnovating, Kaiserslautern. S. 53-66

8. Dupont S, Tafvizi Zavareh M, Zeihsel F, Zink KJ (2020) Entwicklung von Digitalisierungsstrategien. In: Aurich JC, Pier M, Siedler C, Sinnwell C (Hrsg) Bedarfsgerechte Digitalisierung von Produktionsunternehmen. Ein modulares Transformationskonzept als praxisorientierter Ansatz. Synnovating, Kaiserslautern. S. 37-52

9. Ehemann T, Tafvizi Zavareh M, Dupont S, Siedler C, Sinnwell C, Pier M, Prezer A, Jenne F, Batzler F, Zink KJ, Göbel JC (2019) Entwicklung eines Transformationskonzepts zur Digitalisierung in Produktionsunternehmen. In: Bauer, Wilhelm; Stowasser, Sascha; Mütze-Niewöhner, Susanne; Zanker, Claus; Brandl, Karl-Heinz. TransWork - Arbeit in der digitalisierten Welt. Stand der Forschung und Anwendung im BMBF-Förderschwerpunkt. Fraunhofer IAO. Stuttgart120-129 
10. Grimme Landmaschinenfabrik GmbH \& Co. KG (2019) Grimme Gruppe. https://www. grimme.com/de/page/grimmegroup. Zugegriffen: 29. Juli 2019

11. Grimme Landmaschinenfabrik GmbH \& Co. KG (2019). Selbstfahrende Ertetechnik - 22 Tonenn-Bunker I MAXTRON 620. https://www.grimme.com/de/producttypes/selbstfahrendeerntetechnik-ruebe/maxtron-620. Zugegriffen: 2. August 2019

12. Grimme Landmaschinenfabrik GmbH \& Co. KG (2019) Über 150 Jahre Grimme. https:// www.grimme.com/de/page/grimmegroup-history. Zugegriffen: 2. August 2019

13. Hammer W (Hrsg) (1997) Wörterbuch der Arbeitswissenschaft. Begriffe und Definitionen. Hanser, München

14. DIN 69901-5, Januar 2009 (2009) Projektmanagement - Projektmanagementsysteme - Teil 5: Begriffe

15. DIN EN ISO 6385:2016, Dezember 2016 (Dezember 2016) Grundsätze der Ergonomie für die Gestaltung von Arbeitssystemen

16. Junghaus A, Wodrich K, Jeretin-Knopf M, Haas R (2014) Unterstützung von Wartungsarbeiten durch verteilte AR-Assistenzsysteme. In: Weidner R, Redlich T (Hrsg) Erste Transdisziplinäre Konferenz zum Thema Technische Unterstützungssysteme, die die Menschen wirklich wollen. Helmut-Schmidt-Universität, Hamburg, S. 328-338

17. Kotter JP (2007) Leading Change. Why Transformation Efforts Fail. Harvard Business Review (1):3- 11

18. Kuster J, Bachmann C, Huber E, Hubmann M, Lippmann R, Schneider E, Schneider P, Witschi U, Wüst R (2019) Handbuch Projektmanagement. Springer Berlin Heidelberg, Berlin, Heidelberg

19. Maßß W, Pier M, Moser B (2018) Smart Services in der Landwirtschaft. In: Meyer, Kyrill; Klingner, Stephan; Zinke, Christian. Service Engineering. Springer Fachmedien Wiesbaden. Wiesbaden, S.167-181

20. Mascitelli R, Thurnes CM (2015) Mastering lean product development. Geschwindigkeit, Erfolg und Qualität der Produktentwicklung mit Lean-Events maximieren. Synnovating, Kaiserslautern

21. Mont O (2004) Product-service systems. Panacea or myth? Dissertation, Lund University

22. Pfannenberg J (2007) Veränderungskommunikation: Unterstützung von Change-Prozessen. In: Piwinger M, Zerfaß A (Hrsg) Handbuch Unternehmenskommunikation. Gabler Verlag, Wiesbaden

23. Pier M (2015) Standards of mobile Service and predictions for 2020 regarding aftersales, taking an example from Grimme Landmaschinenfabrik GmbH \& Co. KG. 73. International Conference Agricultural Engineering

24. Posselt T, Fuhrmann O, Hofmann B, Danzinger F Industrial Services Excellence Circle. Abschlussbericht 2015, Nürnberg

25. Rupp C, die SOPHISTen (2014) Requirements-Engineering und -Management. Carl Hanser Verlag GmbH \& Co. KG, München

26. Schlick CM, Bruder R, Luczak H (2010) Arbeitswissenschaft. Springer Berlin Heidelberg, Berlin, Heidelberg

27. Siedler C, Dupont S, Ehemann T, Zeihsel F, Sinnwell C, Aurich JC (2020) Vorgehen zur Anwendung des Transformationskonzepts. In: Aurich JC, Pier M, Siedler C, Sinnwell C (Hrsg) Bedarfsgerechte Digitalisierung von Produktionsunternehmen. Ein modulares Transformationskonzept als praxisorientierter Ansatz. Synnovating, Kaiserslautern. S. 67-79

28. Siedler C, Dupont S, Tafvizi Zavareh M, Zeihsel F, Aurich JC (2020) Das Transformationskonzept im Überblick. In: Aurich JC, Pier M, Siedler C, Sinnwell C (Hrsg) Bedarfsgerechte Digitalisierung von Produktionsunternehmen. Ein modulares Transformationskonzept als praxisorientierter Ansatz. Synnovating, Kaiserslautern. S. 17-20 
29. Siedler C, Dupont S, Tafvizi Zavareh M, Zeihsel F, Aurich JC (2020) Reifegradmodell zur Bestimmung des Digitalisierungsgrads. In: Aurich JC, Pier M, Siedler C, Sinnwell C (Hrsg) Bedarfsgerechte Digitalisierung von Produktionsunternehmen. Ein modulares Transformationskonzept als praxisorientierter Ansatz. Synnovating, Kaiserslautern. S. 21-36

30. Siedler C, Sadaune S, Tafvizi Zavareh M, Eigner M, Zink KJ, Aurich JC (2019) Categorizing and selecting digitization technologies for their implementation within different product lifecycle phases. Procedia CIRP 79, S. 274-279. doi:https://doi.org/10.1016/j. procir.2019.02.066

31. Siedler C, Sinnwell C, Aurich JC (2020) Herangehensweise zur pilothaften Konzeptumsetzung. In: Aurich JC, Pier M, Siedler C, Sinnwell C (Hrsg) Bedarfsgerechte Digitalisierung von Produktionsunternehmen. Ein modulares Transformationskonzept als praxisorientierter Ansatz. Synnovating, Kaiserslautern. S. 83-88

32. Sinnwell C, Siedler C, Pier M, Jenne F, Braun S, Schuck M, Zeihsel F, Zink KJ, Göbel JC, Aurich JC (2020) Ausgangssituation und Ziele des Projekts InAsPro. In: Aurich JC, Pier M, Siedler C, Sinnwell C (Hrsg) Bedarfsgerechte Digitalisierung von Produktionsunternehmen. Ein modulares Transformationskonzept als praxisorientierter Ansatz. Synnovating, Kaiserslautern. S. 5-14

33. Stiftung „Mittelstand - Gesellschaft - Verantwortung“ (2019) Kompetenzen der Beschäftigten in 4.0-Prozessen. In: Stiftung „Mittelstand - Gesellschaft - Verantwortung“. Umsetzungshilfen Arbeit 4.0. Künstliche Intelligenz für die produktive und präventive Arbeitsgestaltung nutzen: Hintergrundwissen und Gestaltungsempfehlungen zur Einführung der 4.0-Technologien. Prodis GbR. Heidelberg, S.123-129

34. Stiftung „Mittelstand - Gesellschaft - Verantwortung“ (2019) Lernformen 4.0. In: Stiftung „Mittelstand - Gesellschaft - Verantwortung“. Umsetzungshilfen Arbeit 4.0. Künstliche Intelligenz für die produktive und präventive Arbeitsgestaltung nutzen: Hintergrundwissen und Gestaltungsempfehlungen zur Einführung der 4.0-Technologien. Prodis GbR. Heidelberg, S.137-142

35. Stubbe M, Dupont S (2020) Digitalisierung für Mensch und Organisation im Aftersales. In: Aurich JC, Pier M, Siedler C, Sinnwell C (Hrsg) Bedarfsgerechte Digitalisierung von Produktionsunternehmen. Ein modulares Transformationskonzept als praxisorientierter Ansatz. Synnovating, Kaiserslautern. S. 89-100

36. Tafvizi Zavareh M, Sadaune S, Siedler C, Aurich JC, Zink KJ, Eigner M (2018) A Study on the socio-technical Potentials of industrial Product Development Technologies for future digitized integrated Work Systems. Proceedings of NordDesign 2018

37. TUK - Technische Universität Kaiserslautern (2017) Verbundforschungsprojekt InAsPro. Integrierte Arbeitssystemgestaltung in digitalisierten Produktionsunternehmen. https://www. inaspro.de/. Zugegriffen: 2. August 2019

38. Zink KJ, Kötter W, Longmuß J, Thul MJ (2015) Veränderungsprozesse erfolgreich gestalten. 2. Auflage, Springer Berlin Heidelberg, Berlin, Heidelberg 
Open Access Dieses Kapitel wird unter der Creative Commons Namensnennung 4.0 International Lizenz (http://creativecommons.org/licenses/by/4.0/deed.de) veröffentlicht, welche die Nutzung, Vervielfältigung, Bearbeitung, Verbreitung und Wiedergabe in jeglichem Medium und Format erlaubt, sofern Sie den/die ursprünglichen Autor(en) und die Quelle ordnungsgemäß nennen, einen Link zur Creative Commons Lizenz beifügen und angeben, ob Änderungen vorgenommen wurden.

Die in diesem Kapitel enthaltenen Bilder und sonstiges Drittmaterial unterliegen ebenfalls der genannten Creative Commons Lizenz, sofern sich aus der Abbildungslegende nichts anderes ergibt. Sofern das betreffende Material nicht unter der genannten Creative Commons Lizenz steht und die betreffende Handlung nicht nach gesetzlichen Vorschriften erlaubt ist, ist für die oben aufgeführten Weiterverwendungen des Materials die Einwilligung des jeweiligen Rechteinhabers einzuholen.

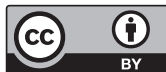

